\title{
Streamline Diffusion Virtual Element Method for Convection-Dominated Diffusion Problems
}

\author{
Yuxia $\mathrm{Li}^{1}$, Cong Xie ${ }^{1,2}$ and Xinglong Feng ${ }^{1, *}$ \\ ${ }^{1}$ College of Mathematics and Systems Science, Xinjiang University, \\ Urumqi 830046, P.R. China. \\ ${ }^{2}$ College of Mathematics and Physics, Hebei University of Architecture, \\ Zhangjiakou, Hebei 075000, P.R. China.
}

Received 23 November 2018; Accepted (in revised version) 24 June 2019.

\begin{abstract}
A novel streamline diffusion form of virtual element method for convectiondominated diffusion problems is studied. The main feature of the method is that the test function in the stabilised term has the adjoint operator-like form $(-\nabla \cdot(K(\mathbf{x}) \nabla v)-\mathbf{b}(\mathbf{x})$. $\nabla v$ ). Unlike the standard VEM, the stabilisation scheme can efficiently avoid nonphysical oscillations. The well-posedness of the problem is also proven and error estimates are provided. Numerical examples show the stability of the method for very large Péclet numbers and its applicability to boundary layer problem.
\end{abstract}

AMS subject classifications: 65N12, 76R99

Key words: Convection dominated diffusion problem, streamline diffusion virtual element method, stabilisation, optimal convergence, boundary layer problem.

\section{Introduction}

Diffusion problems play an important role in various applications, including river and air pollution, fluid flow and fluid heat conduction. It is well known that for convection fields, which are relatively large with respect to the diffusivity, the solutions of the corresponding diffusion problems may have boundary and interior layers. Since standard numerical methods do not work well in such situations, a variety of robust schemes for convectiondominated problems, such as streamline upwind/Petrov-Galerkin formulation [15, 18, 27], residual-free bubbles methods $[12,19]$, discontinuous Galerkin methods [20], finite volume method [25], characteristic finite difference method [26], and multiquadric RBF-FD method [22] have been developed. Recently, Duan et al. [17] introduced a numerical method with deterministic and explicit stabilisation parameter for the reaction-convectiondiffusion equations having a large reaction coefficient. For equations with arbitrary magnitude of reaction and diffusion, Hsieh and Yang [21] proposed a new method with a test

${ }^{*}$ Corresponding author. Email addresses: Iy x29cherish@163.com (Y. Li), xiecong121@163.com (C. Xie), fxlmath@xju.edu.cn (X. Feng) 
function in the stabilising term. We also note the virtual element method (VEM) in [1,3-5]. It can be viewed as the evolution of the mimetic finite difference (MFD) method $[13,23]$ or as an extension of the traditional finite element method (FEM) to polygons or polyhedron elements [28]. VEM was applied to various problems, such as plate bending [14], elasticity [6], Stokes [2] and Navier-Stokes equations [7], simplified friction problem [29]. The method performs well in geometrically complex domains [8] and with badly shaped polygonal elements [10]. Cangiani et al. [16] first discussed a non-consistent SUPG-VEM formulation for the convection-dominated diffusion problem, Benedetto et al. [9] established a consistent VEM-SUPG formulation, and Berrone et al. [11] developed a SUPG stabilisation for the nonconforming VEM. The robustness of a priori error estimates for these methods can be proved for high Péclet numbers. This shows the efficiency of the SUPG stabilisation.

The present work is motivated by the ideas of [21] and aims to construct a new kind of streamline diffusion VEM for convection-dominated diffusion problems. We use a test function in the stabilisation term in the adjoint-operator-like form $-\nabla \cdot(K \nabla v)-\mathbf{b} \cdot \nabla v$. In contrast to others SUPG formulations, the stabilisation parameter $\tau$ has a simple representation and can be easily computed for each element. In order to comply to VEMs with implicit basis functions, we compute the projections of the stabilisation terms onto polynomial spaces. The well-posedness and optimal error estimates of the stabilisation scheme are established by introducing an error norm. Numerical results show that the stabilisation scheme can efficiently treat boundary layer problems and avoid nonphysical oscillations.

The paper is organised as follows. In Section 2, we introduce a model problem and consider a streamline diffusion virtual element method. A priori error estimates are presented by Theorem 3.2 in Section 3, whereas Section 4 contains some results of numerical tests to support the theoretical findings. Finally, some conclusions are drawn in the last section.

\section{Streamline Diffusion VEM for a Model Problem}

We consider the streamline diffusion virtual element approximations for the following Dirichlet boundary value convection-dominated diffusion problem:

$$
\begin{array}{cl}
-\nabla \cdot(K(\mathbf{x}) \nabla u)+\mathbf{b}(\mathbf{x}) \cdot \nabla u=f(\mathbf{x}), & \text { in } \Omega, \\
u(\mathbf{x})=0, & \text { on } \partial \Omega,
\end{array}
$$

where $\Omega \subset \mathbb{R}^{2}$ is an open bounded convex polygonal domain with the boundary $\partial \Omega$, $u$ the physical quantity of interest, $K(\mathbf{x}) \in L^{\infty}(\Omega)$ the symmetric diffusion tensor, $\mathbf{b}(\mathbf{x}) \in(C(\Omega))^{2}$ the convection field such that $\nabla \cdot \mathbf{b}(\mathbf{x})=0$ in $\Omega$ and $f(\mathbf{x})$ a given source function.

Let $(\cdot, \cdot)$ and $\|\cdot\|$ refer to $L^{2}(\Omega)$ scalar products and norm, respectively, and if $D$ is a subdomain of $\Omega$, we write $(\cdot, \cdot)_{D}$ and $\|\cdot\|_{D}$ for the corresponding $L^{2}(D)$ scalar product and the norm. Besides, $\|\cdot\|_{m}$ and $|\cdot|_{m}$ are the $H^{m}(\Omega)$ norm and semi-norm and $\|\cdot\|_{m, D}$ and $|\cdot|_{m, D}$ the $H^{m}(D)$ norm and semi-norm. Let $\mathbb{P}_{k}(D)$ be the space of polynomials of degree at most $k$ on $D$. The dimension $N_{P}$ of $\mathbb{P}_{k}(D)$ is $(1 / 2)(k+1)(k+2)$. 


\subsection{Virtual element method}

Let $\left\{\mathscr{T}_{h}\right\}_{0<h \leq 1}$ be a family of polygons such that $\bar{\Omega}=\bigcup_{E \in \mathscr{T}_{h}} E$, where $E$ are non-overlapping star-shaped polygonal elements and let $h:=\max \left\{h_{E}, E \in \mathscr{T}_{h}\right\}$, where $h_{E}$ is the diameter of $E$. The number of edges of an element $E$ is denoted by $N^{E}$. We always assume that the family $\left\{\mathscr{T}_{h}\right\}_{0<h \leq 1}$ is shape regular [5], i.e. there is a constant $\rho>0$ such that

- If $e \in \partial E$, then $h_{e} \geq \rho h_{E}$ for any $E$.

- If $P, Q$ are nodes of $E$, then $\|P-Q\| \geq \rho h_{E}$.

Besides, we also use the notations

$$
K_{E}:=\sup _{\mathbf{x} \in E} K(\mathbf{x}), \quad K_{E}^{V}:=\inf _{\mathbf{x} \in E} K(\mathbf{x}), \quad b_{E}:=\sup _{\mathbf{x} \in E}\|\mathbf{b}(\mathbf{x})\|_{\mathbb{R}^{2}} .
$$

In this work, we deal with the lowest virtual function space - i.e. we assume that $k=1$. Following [3], for any $E \in \mathscr{T}_{h}$ we consider the local space

$$
W_{h}(E):=\left\{w \in H^{1}(E):\left.w_{h}\right|_{\partial E} \in B(\partial E),\left.\Delta w_{h}\right|_{E} \in \mathbb{P}_{-1}(E):=0\right\}
$$

where

$$
B(\partial E):=\left\{w_{h} \in C^{0}(\partial E):\left.w_{h}\right|_{e} \in \mathbb{P}_{1}(E), \forall e \subset \partial E\right\} .
$$

Let $\left(x_{E}, y_{E}\right)$ be the barycenter of $E$ and

$$
\mathscr{M}(E):=\left\{m_{1}(x, y):=1, m_{2}(x, y):=\frac{x-x_{E}}{h_{E}}, m_{3}(x, y):=\frac{y-y_{E}}{h_{E}}\right\}
$$

be the barycentric polynomials on $E$. The cardinality $N_{P}$ of this basis is 3 , the same as the dimension of the space $\mathbb{P}_{1}(E)$.

Definition 2.1 (cf. Beirão Da Veiga et al. [4]). The $H^{1}$-Ritz operator $\Pi^{\nabla}: W_{h}(E) \rightarrow \mathbb{P}_{1}(E)$ is defined by

$$
\left(\nabla\left(\Pi^{\nabla} w_{h}-w_{h}\right), \nabla p\right)_{0, E}=0 \quad \text { for all } \quad w_{h} \in W_{h}(E), \quad p \in \mathbb{P}_{1}(E), \quad \overline{\Pi^{\nabla} w_{h}}=\overline{w_{h}}
$$

where

$$
\overline{w_{h}}:=\frac{1}{N_{E}} \sum_{i=1}^{N_{E}} w_{h}\left(\mathrm{v}_{i}\right)
$$

is the average value of $w_{h}$ at the vertices of $E$.

The second condition in the above definition is needed for the uniqueness of the projection operator.

The local $V_{h}(E)$ and the global $V_{h}(\Omega)$ virtual element spaces can be defined by

$$
\begin{aligned}
& V_{h}(E)=\left\{v_{h}: v_{h} \in B(\partial E), \Delta v_{h} \in P_{1}(E),\left(v_{h}-\Pi^{\nabla} v_{h}, q\right)=0, \forall q \in P_{1}(E)\right\}, \\
& V_{h}(\Omega)=\left\{v_{h} \in H_{0}^{1}(\Omega):\left.v_{h}\right|_{E} \in V_{h}(E), \forall E \in \mathscr{T}_{h}\right\} .
\end{aligned}
$$


Definition 2.2. The $L^{2}$-Ritz operator $\Pi^{0}: V_{h}(E) \rightarrow \mathbb{P}_{1}(E)$ is defined by

$$
\left(\left(\Pi^{0} w_{h}-w_{h}\right), p\right)_{0, E}=0, \quad \forall w_{h} \in V_{h}(E), \quad p \in \mathbb{P}_{1}(E) .
$$

Remark 2.1. For linear VEMs, one has $\Pi^{\nabla}=\Pi^{0}$, cf. $[1,4]$.

The degrees of freedom for $V_{h}$ are the values of $v_{h}$ at all vertexes in the domain. Let $\operatorname{dof}_{i}: V_{h}(E) \rightarrow R, i=1,2, \ldots, N^{E}$ be the operator, which assign to any $\left(v_{h}\right) \in V_{h}(E)$ the $i$-th degree of freedom of $v_{h}$. Besides, let $\phi_{i} \in V_{h}(E)$ be the basis functions defined by

$$
\operatorname{dof}_{i}\left(\phi_{j}\right):=\delta_{i j}, \quad i, j=1,2, \ldots, N^{E} .
$$

Thus we have $v_{h}=\sum_{i=1}^{N^{E}} \operatorname{dof}_{i}\left(v_{h}\right) \phi_{i}$ for all $v_{h} \in V_{h}(E)$.

\subsection{Streamline diffusion VEM formulation}

We now consider the following stabilised streamline diffusion VEM for the model problem (2.1): Find a function $u \in H_{0}^{1}(\Omega)$ such that

$$
A_{s}(u, v):=L_{s}(v) \text { for all } v \in H_{0}^{1}(\Omega),
$$

where $L_{s}$ and $A_{s}$ are, respectively, linear and bilinear forms defined by

$$
L_{s}(v):=(f, v)-\sum_{E \in \mathscr{T}_{h}} \tau_{E}(f,-\nabla \cdot(K \nabla v)-\mathbf{b} \cdot \nabla v)_{E},
$$

and

$$
\begin{aligned}
& A_{s}(u, v):=a(u, v)+b(u, v)+d(u, v), \\
& a(u, v)=(K \nabla u \cdot \nabla v)+\sum_{E \in \mathscr{T}_{h}} \tau_{E}(\mathbf{b} \cdot \nabla u, \mathbf{b} \cdot \nabla v)_{E}-\sum_{E \in \mathscr{T}_{h}} \tau_{E}(\nabla \cdot(K \nabla u), \nabla \cdot(K \nabla v))_{E}, \\
& b(u, v)=(\mathbf{b} \cdot \nabla u, v), \\
& d(u, v)=\sum_{E \in \mathscr{T}_{h}} \tau_{E}(\mathbf{b} \cdot \nabla u, \nabla \cdot(K \nabla v))_{E}-\sum_{E \in \mathscr{T}_{h}} \tau_{E}(\nabla \cdot(K \nabla u), \mathbf{b} \cdot \nabla v)_{E} .
\end{aligned}
$$

The stabilisation parameter $\tau_{E}$ is given by

$$
\tau_{E}=\frac{h_{E}^{2}}{8 K_{E}+2 b_{E} h_{E}}
$$

and satisfies the inequality $\tau_{E} \leq\left(C_{E} h_{E}^{2}\right) /\left(8 K_{E}\right)$, where $C_{E}$ is the largest possible constant in the inverse inequality

$$
C_{E} h_{E}^{2}\left\|\nabla \cdot\left(K \nabla v_{h}\right)\right\|_{E}^{2} \leq\left\|K \nabla v_{h}\right\|_{E}^{2} \quad \text { for all } \quad v_{h} \in V_{h}(E)
$$


noted in [9]. Moreover, $\tau_{E}>0$ and $\tau_{E} \leq h_{E} /\left(2 b_{E}\right)$. We emphasize that the main difference of our work from the stabilisation method in [9] is that the test function in the stabilisation term has an adjoint-operator-like form, viz. $-\nabla \cdot(K \nabla v)-\mathbf{b} \cdot \nabla v$.

Now we define the discrete bilinear form $A_{s, h}: V_{h} \times V_{h} \longrightarrow \mathbb{R}$ by

$$
A_{s, h}\left(u_{h}, v_{h}\right):=a_{h}\left(u_{h}, v_{h}\right)+b_{h}\left(u_{h}, v_{h}\right)+d_{h}\left(u_{h}, v_{h}\right) \text { for all } u_{h}, v_{h} \in V_{h},
$$

where

$$
\begin{aligned}
a_{h}\left(u_{h}, v_{h}\right):= & \left(K \nabla\left(\Pi^{\nabla} u_{h}\right), \nabla\left(\Pi^{\nabla} v_{h}\right)\right)+\sum_{E \in \mathscr{T}_{h}} \tau_{E}\left(\mathbf{b} \cdot \nabla\left(\Pi^{\nabla} u_{h}\right), \mathbf{b} \cdot \nabla\left(\Pi^{\nabla} v_{h}\right)\right)_{E} \\
& -\sum_{E \in \mathscr{T}_{h}} \tau_{E}\left(\nabla \cdot\left(K \nabla\left(\Pi^{\nabla} u\right)\right), \nabla \cdot\left(K \nabla\left(\Pi^{\nabla} v\right)\right)\right)_{E} \\
& +\sum_{E \in \mathscr{T}_{h}}\left(K_{E}+\tau_{E} b_{E}^{2}\right) S_{a}^{E}\left(\left(I-\Pi^{\nabla}\right) u_{h},\left(I-\Pi^{\nabla}\right) v_{h}\right), \\
b_{h}\left(u_{h}, v_{h}\right):= & \left(\mathbf{b} \cdot \nabla\left(\Pi^{\nabla} u_{h}\right), \Pi^{0} v_{h}\right), \\
d_{h}\left(u_{h}, v_{h}\right):= & \sum_{E \in \mathscr{T}_{h}} \tau_{E}\left(\mathbf{b} \cdot \nabla\left(\Pi^{\nabla} u_{h}\right), \nabla \cdot\left(K \nabla\left(\Pi^{\nabla} v_{h}\right)\right)\right)_{E} \\
& -\sum_{E \in \mathscr{T}_{h}} \tau_{E}\left(\nabla \cdot\left(K \nabla\left(\Pi^{\nabla} u_{h}\right)\right), \mathbf{b} \cdot \nabla\left(\Pi^{\nabla} v_{h}\right)\right)_{E}
\end{aligned}
$$

and $S_{a}^{E}\left(\left(I-\Pi^{\nabla}\right) u_{h},\left(I-\Pi^{\nabla}\right) v_{h}\right)$ is the stabilised term approximating the inner product $\left(\nabla\left(u_{h}-\Pi^{\nabla} u_{h}\right), \nabla\left(v_{h}-\Pi^{\nabla} v_{h}\right)\right)$. Following [9], we impose the condition

$$
S_{a}^{E}\left(v_{h}, v_{h}\right) \sim\left\|\nabla v_{h}\right\|_{E}^{2} \quad \text { for all } \quad v_{h} \in \operatorname{ker} \Pi^{\nabla},
$$

which guarantees the stability in the space $V_{h}(E) / \mathbb{P}_{1}(E)$. Consequently,

$$
S_{a}^{E}\left(\left(I-\Pi^{\nabla}\right) u_{h},\left(I-\Pi^{\nabla}\right) v_{h}\right) \leq\left\|\nabla\left(u_{h}-\Pi^{\nabla} u_{h}\right)\right\|_{E}\left\|\nabla\left(v_{h}-\Pi^{\nabla} v_{h}\right)\right\|_{E} .
$$

The operator $S_{a}^{E}$ is chosen as in [4,9] - i.e.

$$
S_{a}^{E}\left(\left(I-\Pi^{\nabla}\right) \phi_{i},\left(I-\Pi^{\nabla}\right) \phi_{j}\right)=\sum_{i=1}^{N_{E}} \operatorname{dof}_{i}\left(\left(I-\Pi^{\nabla}\right) \phi_{i}\right) \operatorname{dof}_{i}\left(\left(I-\Pi^{\nabla}\right) \phi_{j}\right),
$$

and $L_{s}$ is discretised as follows:

$$
L_{s, h}\left(v_{h}\right)=\left(f, \Pi^{0} v_{h}\right)-\sum_{E \in \mathscr{T}_{h}} \tau_{E}\left(f,-\nabla \cdot\left(K \nabla\left(\Pi^{\nabla} v_{h}\right)\right)-\mathbf{b} \cdot \nabla\left(\Pi^{\nabla} v_{h}\right)\right)_{E} \quad \text { for all } \quad v_{h} \in V_{h}
$$

Thus an approximate solution $u_{h} \in V_{h}(\Omega)$ of the problem (2.1) is determined from the following equation:

$$
A_{s, h}\left(u_{h}, v_{h}\right)=L_{s, h}\left(v_{h}\right) \quad \text { for all } \quad v_{h} \in V_{h}(\Omega)
$$




\section{Error Analysis}

We first consider the discretisation errors.

Lemma 3.1 (cf. Beirão Da Veiga et al. [5]). If $E \in \mathscr{T}_{h}$ and $w \in H^{2}(E)$, then

$$
\begin{array}{ll}
\left\|w-\Pi^{0} w\right\|_{m, E} \leq C h_{E}^{s-m}|w|_{s, E}, & m, s \in \mathbb{N}, \quad m \leq s \leq 2, \\
\left\|w-\Pi^{\nabla} w\right\|_{m, E} \leq C h_{E}^{s-m}|w|_{s, E}, & m, s \in \mathbb{N}, \quad m \leq s \leq 2, \quad s \geq 1,
\end{array}
$$

where $\Pi^{\nabla} w$ and $\Pi^{0} w \in \mathbb{P}_{1}(E)$ are the projections of $w$ to the projection space.

Here and in what follows $C$ is a positive constant, which may depend on the mesh regularity and take different values in different situations.

Lemma 3.2. If $K_{E} \ll h_{E}$, then for any sufficiently regular function $\varphi$ and for any $E$ in $\mathscr{T}_{h}$ the following inequality holds:

$$
a_{h}\left(\varphi, v_{h}\right) \leq C \max _{E \in \mathscr{T}_{h}} \frac{K_{E}+\tau_{E} b_{E}^{2}}{K_{E}^{V}}\|\sqrt{K} \nabla \varphi\|\left\|\sqrt{K} \nabla v_{h}\right\| .
$$

Moreover, if $K \in W_{\infty}^{1}(\Omega)$ and $\mathbf{b} \in\left[W_{\infty}^{1}(\Omega)\right]^{2}$, then

$$
\left|a\left(\varphi, v_{h}\right)-a_{h}\left(\varphi, v_{h}\right)\right| \leq C \max _{E \in \mathscr{T}_{h}} \frac{\|K\|_{W_{\infty}^{1}}+\|\mathbf{b}\|_{W_{\infty}^{1}}+1 / b_{E}}{\sqrt{K_{E}^{V}}} h\|\varphi\|_{2}\left\|\sqrt{K} \nabla v_{h}\right\| .
$$

Proof. We start with the estimate (3.3). Relations (2.2),(2.4), the Cauchy-Schwarz inequality and the continuity of the projectors yield

$$
\begin{aligned}
a_{h}^{E}\left(\varphi, v_{h}\right)= & \left(K \nabla\left(\Pi^{\nabla} \varphi\right), \nabla\left(\Pi^{\nabla} v_{h}\right)\right)_{E}+\tau_{E}\left(\mathbf{b} \cdot \nabla\left(\Pi^{\nabla} \varphi\right), \mathbf{b} \cdot \nabla\left(\Pi^{\nabla} v_{h}\right)\right)_{E} \\
& -\tau_{E}\left(\nabla \cdot\left(K \nabla\left(\Pi^{\nabla} \varphi\right)\right), \nabla \cdot\left(K \nabla\left(\Pi^{\nabla} v_{h}\right)\right)\right)_{E} \\
& +\left(K_{E}+\tau_{E} b_{E}^{2}\right) S_{a}^{E}\left(\left(I-\Pi^{\nabla}\right) \varphi,\left(I-\Pi^{\nabla}\right) v_{h}\right)_{E} \\
\leq & \left\|K \nabla\left(\Pi^{\nabla} \varphi\right)\right\|_{E}\left\|\nabla\left(\Pi^{\nabla} v_{h}\right)\right\|_{E}+\tau_{E}\left\|\mathbf{b} \cdot \nabla\left(\Pi^{\nabla} \varphi\right)\right\|_{E}\left\|\mathbf{b} \cdot \nabla\left(\Pi^{\nabla} v_{h}\right)\right\|_{E} \\
& +\tau_{E}\left\|\nabla \cdot\left(K \nabla\left(\Pi^{\nabla} \varphi\right)\right)\right\|_{E}\left\|\nabla \cdot\left(K \nabla\left(\Pi^{\nabla} v_{h}\right)\right)\right\|_{E} \\
& +\left(K_{E}+\tau_{E} b_{E}^{2}\right)\left\|\nabla\left(I-\Pi^{\nabla}\right) \varphi\right\|_{E}\left\|\nabla\left(I-\Pi^{\nabla}\right) v_{h}\right\|_{E} \\
\leq & \left(K_{E}+\tau_{E} b_{E}^{2}\right)\left(\left\|\nabla\left(\Pi^{\nabla} \varphi\right)\right\|_{E}\left\|\nabla\left(\Pi^{\nabla} v_{h}\right)\right\|_{E}+\left\|\nabla\left(I-\Pi^{\nabla}\right) \varphi\right\|_{E}\right. \\
& \left.\times\left\|\nabla\left(I-\Pi^{\nabla}\right) v_{h}\right\|_{E}\right)+\frac{\tau_{E}}{C_{E} h_{E}^{2}}\left\|K \nabla\left(\Pi^{\nabla} \varphi\right)\right\|_{E}\left\|K \nabla\left(\Pi^{\nabla} v_{h}\right)\right\|_{E} \\
\leq & C \frac{K_{E}+\tau_{E} b_{E}^{2}}{K_{E}^{V}}\|\sqrt{K} \nabla \varphi\|_{E}\left\|\sqrt{K} \nabla v_{h}\right\|_{E} \quad \text { for all } \quad E \in \mathscr{T}_{h} .
\end{aligned}
$$

It follows from the definition of the operators $\Pi^{0}$ and $\Pi^{\nabla}$ that

$$
\left(K \nabla \varphi, \nabla\left(\Pi^{\nabla} v_{h}\right)\right)_{E}=\left(\Pi^{0}(K \nabla \varphi), \nabla\left(\Pi^{\nabla} v_{h}\right)\right)_{E},
$$




$$
\left(\Pi^{0}(K \nabla \varphi), \nabla\left(\Pi^{\nabla} v_{h}\right)\right)_{E}=\left(\Pi^{0}(K \nabla \varphi), \nabla v_{h}\right)_{E}
$$

and since $\Pi^{\nabla}=\Pi^{0}$, then

$$
\begin{aligned}
& \left(K \nabla \varphi, \nabla\left(\Pi^{\nabla} v_{h}\right)\right)_{E}=\left(\Pi^{\nabla}(K \nabla \varphi), \nabla v_{h}\right)_{E}, \\
& \left(\mathbf{b b}^{T} \nabla \varphi, \nabla\left(\Pi^{\nabla} v_{h}\right)\right)_{E}=\left(\Pi^{\nabla}\left(\mathbf{b b}^{T} \nabla \varphi\right), \nabla v_{h}\right)_{E} .
\end{aligned}
$$

Concerning (3.4), we add and subtract $\left(K \nabla \varphi, \nabla\left(\Pi^{\nabla} v_{h}\right)\right)_{E}$ and $\left(\mathbf{b b}^{T} \nabla \varphi, \nabla\left(\Pi^{\nabla} v_{h}\right)\right)_{E}$ and using the triangle inequality, for any $E \in \mathscr{T}_{h}$ we obtain

$$
\begin{aligned}
& \left|a^{E}\left(\varphi, v_{h}\right)-a_{h}^{E}\left(\varphi, v_{h}\right)\right| \\
\leq & \left|\left(K \nabla \varphi-K \nabla\left(\Pi^{\nabla} \varphi_{h}\right), \nabla\left(\Pi^{\nabla} v_{h}\right)\right)_{E}\right| \\
& +\left|\left(K \nabla \varphi-\Pi^{\nabla}(K \nabla \varphi), \nabla v_{h}\right)_{E}\right| \\
& +\tau_{E}\left(\left|\left(\mathbf{b b}^{T} \nabla \varphi-\mathbf{b b}^{T} \nabla\left(\Pi^{\nabla} \varphi\right), \nabla\left(\Pi^{\nabla} v_{h}\right)\right)_{E}\right|\right. \\
& \left.+\left|\left(\mathbf{b b}^{T} \nabla \varphi-\Pi^{\nabla}\left(\mathbf{b b}^{T} \nabla \varphi\right), \nabla v_{h}\right)_{E}\right|\right) \\
& +\tau_{E}\left(\left|\left(\nabla \cdot\left(K\left(\nabla \varphi-\nabla\left(\Pi^{\nabla} \varphi\right)\right)\right), \nabla \cdot\left(K \nabla v_{h}\right)\right)_{E}\right|\right. \\
& \left.+\left|\left(\nabla \cdot\left(K \nabla\left(\Pi^{\nabla} \varphi\right)\right), \nabla \cdot K\left(\nabla v_{h}-\nabla\left(\Pi^{\nabla} v_{h}\right)\right)\right)_{E}\right|\right) \\
& +\left(K_{E}+\tau_{E} b_{E}^{2}\right)\left|S_{a}^{E}\left(\left(I-\Pi^{\nabla}\right) \varphi,\left(I-\Pi^{\nabla}\right) v_{h}\right)\right|,
\end{aligned}
$$

where the identities

$$
\begin{aligned}
& \left(K \nabla \varphi, \nabla\left(\Pi^{\nabla} v_{h}\right)\right)_{E}=\left(\Pi^{\nabla}(K \nabla \varphi), \nabla v_{h}\right)_{E}, \\
& \left(\mathbf{b b}^{T} \nabla \varphi, \nabla\left(\Pi^{\nabla} v_{h}\right)\right)_{E}=\left(\Pi^{\nabla}\left(\mathbf{b b}^{T} \nabla \varphi\right), \nabla v_{h}\right)_{E}
\end{aligned}
$$

are used. Every row in the right-hand side will be estimated separately. The CauchySchwarz inequality and (3.1) imply

$$
\begin{aligned}
& \left|\left(K\left(\nabla \varphi-\nabla\left(\Pi^{\nabla} \varphi\right)\right), \nabla\left(\Pi^{\nabla} v_{h}\right)\right)_{E}\right|+\left|\left(K \nabla \varphi-\Pi^{\nabla}(K \nabla \varphi), \nabla v_{h}\right)_{E}\right| \\
\leq & C\left(K_{E}\left\|\nabla \varphi-\nabla\left(\Pi^{\nabla} \varphi\right)\right\|_{E}\left\|\nabla v_{h}\right\|_{E}+\left\|K \nabla \varphi-\Pi^{\nabla}(K \nabla \varphi)\right\|_{E}\left\|\nabla v_{h}\right\|_{E}\right) \\
\leq & C \frac{1}{\sqrt{K_{E}^{V}}}\left(K_{E} h_{E}|\varphi|_{2, E}\left\|\sqrt{K} \nabla v_{h}\right\|_{E}+|K \nabla \varphi|_{1, E}\left\|\sqrt{K} \nabla v_{h}\right\|_{E}\right) \\
\leq & C \frac{\|K\|_{W_{\infty}^{1}}}{\sqrt{K_{E}^{V}}} h_{E}\|\varphi\|_{2, E}\left\|\sqrt{K} \nabla v_{h}\right\|_{E} .
\end{aligned}
$$

Next we use the Cauchy-Schwarz inequality, the inequality (3.1) and the continuity of $\Pi^{\nabla}$, so that

$$
\tau_{E}\left(\left|\left(\mathbf{b b}^{T} \nabla \varphi-\mathbf{b b}^{T} \nabla\left(\Pi^{\nabla} \varphi\right), \nabla\left(\Pi^{\nabla} v_{h}\right)\right)_{E}\right|+\left|\left(\mathbf{b b}^{T} \nabla \varphi-\Pi^{\nabla}\left(\mathbf{b b}^{T} \nabla \varphi\right), \nabla v_{h}\right)_{E}\right|\right)
$$




$$
\begin{aligned}
& \leq \tau_{E}\left(\left\|\mathbf{b b}^{T} \nabla \varphi-\mathbf{b b}^{T} \nabla\left(\Pi^{\nabla} \varphi\right)\right\|_{E}\left\|\nabla\left(\Pi^{\nabla} v_{h}\right)\right\|_{E}+\left\|\mathbf{b b}^{T} \nabla \varphi-\Pi^{\nabla}\left(\mathbf{b b}^{T} \nabla \varphi\right)\right\|_{E}\left\|\nabla v_{h}\right\|_{E}\right) \\
& \leq C \tau_{E}\left(b_{E}^{2}\left\|\nabla \varphi-\nabla\left(\Pi^{\nabla} \varphi\right)\right\|_{E}\left\|\nabla v_{h}\right\|_{E}+\left|\mathbf{b b}^{T} \nabla \varphi\right|_{1, E}\left\|\nabla v_{h}\right\|_{E}\right) \\
& \leq C \frac{\|b\|_{W_{\infty}^{1}}^{1}}{\sqrt{K_{E}^{V}}} h_{E}\|\varphi\|_{2, E}\left\|\sqrt{K} \nabla v_{h}\right\|_{E} .
\end{aligned}
$$

On the next step, we recall (2.2) to obtain

$$
\begin{aligned}
& \tau_{E}\left(\left|\left(\nabla \cdot\left(K\left(\nabla \varphi-\nabla\left(\Pi^{\nabla} \varphi\right)\right)\right), \nabla \cdot\left(K \nabla v_{h}\right)\right)_{E}\right|\right. \\
& \left.+\left|\left(\nabla \cdot\left(K \nabla\left(\Pi^{\nabla} \varphi\right)\right), \nabla \cdot K\left(\nabla v_{h}-\nabla\left(\Pi^{\nabla} v_{h}\right)\right)\right)_{E}\right|\right) \\
\leq & C \frac{\tau_{E}\|K\|_{W_{\infty}^{1}}^{2}}{h_{E}^{2}}\left(\left\|\nabla \varphi-\nabla\left(\Pi^{\nabla} \varphi\right)\right\|_{E}\left\|\nabla v_{h}\right\|_{E}+\left\|\nabla\left(\Pi^{\nabla} \varphi\right)\right\|_{E}\left\|\nabla v_{h}-\nabla\left(\Pi^{\nabla} v_{h}\right)\right\|_{E}\right) \\
\leq & C \frac{\tau_{E}\|K\|_{W_{\infty}^{1}}^{2}}{h_{E}^{2}}\left(\|\nabla \varphi\|_{E}\left\|\nabla v_{h}\right\|_{E}+\left\|\nabla\left(\Pi^{\nabla} \varphi\right)\right\|_{E}\left\|\nabla v_{h}\right\|_{E}\right) \\
\leq & C \frac{\|K\|_{W_{\infty}^{1}}^{2}}{b_{E} h_{E} \sqrt{K_{E}^{V}}}\|\varphi\|_{2, E}\left\|\sqrt{K} \nabla v_{h}\right\|_{E} \leq C \frac{1 / b_{E}}{\sqrt{K_{E}^{V}}} h_{E}\|\varphi\|_{2, E}\left\|\sqrt{K} \nabla v_{h}\right\|_{E} .
\end{aligned}
$$

Finally, the continuity of $\Pi^{\nabla}$ and (3.2) yields

$$
\begin{aligned}
& K_{E}\left\|\nabla\left(\varphi-\Pi^{\nabla} \varphi\right)\right\|_{E}\left\|\nabla\left(v_{h}-\Pi^{\nabla} v_{h}\right)\right\|_{E} \leq C \frac{K_{E}}{\sqrt{K_{E}^{V}}} h_{E}|\varphi|_{2, E}\left\|\sqrt{K} \nabla v_{h}\right\|_{E}, \\
& \tau_{E} b_{E}^{2}\left\|\nabla\left(\varphi-\Pi^{\nabla} \varphi\right)\right\|_{E}\left\|\nabla\left(v_{h}-\Pi^{\nabla} v_{h}\right)\right\|_{E} \leq C \frac{b_{E}}{\sqrt{K_{E}^{V}}} h_{E}^{2}|\varphi|_{2, E}\left\|\sqrt{K} \nabla v_{h}\right\|_{E},
\end{aligned}
$$

and consequently (3.4).

Lemma 3.3. For any $E \in \mathscr{T}_{h}$ and any sufficiently regular function $\varphi$ we have

$$
b_{h}\left(\varphi, v_{h}\right) \leq C \max _{E \in \mathscr{T}_{h}} \frac{b_{E}}{\sqrt{K_{E}^{V}}}\|\sqrt{K} \nabla \varphi\|\left\|v_{h}\right\| .
$$

Moreover, if $\mathbf{b}(\boldsymbol{x}) \in\left[W_{\infty}^{1}(\Omega)\right]^{2}$, then

$$
\left|b\left(\varphi, v_{h}\right)-b_{h}\left(\varphi, v_{h}\right)\right| \leq C \max _{E \in \mathscr{T}_{h}}\|\mathbf{b}\|_{W_{\infty}^{1}(E)} h^{2}\|\varphi\|_{2}\left\|v_{h}\right\|_{1} .
$$

Proof. The Cauchy-Schwarz inequality and the continuity of $\Pi^{\nabla}$ and $\Pi^{0}$ lead to the estimate (3.5) - viz.

$$
b_{h}^{E}\left(\varphi, v_{h}\right)=\left(\mathbf{b} \cdot \nabla\left(\Pi^{\nabla} \varphi\right), \Pi^{0} v_{h}\right)_{E}
$$




$$
\leq b_{E}\left(\left\|\nabla\left(\Pi^{\nabla} \varphi\right)\right\|_{E}\left\|\Pi^{0} v_{h}\right\|_{E}\right) \leq C \frac{b_{E}}{\sqrt{K_{E}^{V}}}\|\sqrt{K} \nabla \varphi\|_{E}\left\|v_{h}\right\|_{E} .
$$

On the other hand, for any $p \in \mathbb{P}_{1}(E)$ we have

$$
\begin{gathered}
\left(\nabla \Pi^{\nabla} \varphi, \nabla p\right)=(\nabla \varphi, \nabla p)=\left(\frac{\partial \varphi}{\partial x_{1}}, \frac{\partial p}{\partial x_{1}}\right)+\left(\frac{\partial \varphi}{\partial x_{2}}, \frac{\partial p}{\partial x_{2}}\right) \\
=\left(\Pi^{\nabla} \frac{\partial \varphi}{\partial x_{1}}, \frac{\partial p}{\partial x_{1}}\right)+\left(\Pi^{\nabla} \frac{\partial \varphi}{\partial x_{2}}, \frac{\partial p}{\partial x_{2}}\right)=\left(\left(\Pi^{\nabla} \frac{\partial \varphi}{\partial x_{1}}, \Pi^{\nabla} \frac{\partial \varphi}{\partial x_{2}}\right), \nabla p\right),
\end{gathered}
$$

so that

$$
\nabla \Pi^{\nabla} \varphi=\left(\Pi^{\nabla} \frac{\partial \varphi}{\partial x_{1}}, \Pi^{\nabla} \frac{\partial \varphi}{\partial x_{2}}\right) .
$$

Considering the difference $b^{E}\left(\varphi, v_{h}\right)-b_{h}^{E}\left(\varphi, v_{h}\right)$, for any $E \in \mathscr{T}_{h}$ we have

$$
\begin{aligned}
& \left|b^{E}\left(\varphi, v_{h}\right)-b_{h}^{E}\left(\varphi, v_{h}\right)\right| \\
= & \left|\left(\mathbf{b} \cdot \nabla \varphi, v_{h}\right)_{E}-\left(\mathbf{b} \cdot \nabla\left(\Pi^{\nabla} \varphi\right), \Pi^{0} v_{h}\right)_{E}\right| \\
\leq & \left|\left(\mathbf{b} \cdot\left(\nabla \varphi-\nabla\left(\Pi^{\nabla} \varphi\right)\right), v_{h}\right)_{E}+\left(\mathbf{b} \cdot \nabla\left(\Pi^{\nabla} \varphi\right),\left(v_{h}-\Pi^{0} v_{h}\right)\right)_{E}\right| \\
\leq & \left|\left(\mathbf{b} \cdot\left(\nabla \varphi-\nabla\left(\Pi^{\nabla} \varphi\right)\right), v_{h}\right)_{E}\right|+\left|\left(\mathbf{b} \cdot \nabla\left(\Pi^{\nabla} \varphi\right),\left(v_{h}-\Pi^{0} v_{h}\right)\right)_{E}\right| \\
= & \sum_{i=1}^{2}\left(\left(\frac{\partial \varphi}{\partial x_{i}}-\Pi^{\nabla} \frac{\partial \varphi}{\partial x_{i}}, \mathbf{b}_{i} v_{h}\right)_{E}+\left(\mathbf{b}_{i} \Pi^{\nabla} \frac{\partial \varphi}{\partial x_{i}}, v_{h}-\Pi^{0} v_{h}\right)_{E}\right) .
\end{aligned}
$$

It follows from Definition 2.1 that

$$
\left(\frac{\partial \varphi}{\partial x_{i}}-\Pi^{\nabla} \frac{\partial \varphi}{\partial x_{i}}, \Pi^{\nabla}\left(\mathbf{b}_{i} v_{h}\right)\right)=0 .
$$

Hence, for both $i=1$ and $i=2$ we can evaluate the first residue in the sum in the right-hand side of (3.7) as follows

$$
\begin{aligned}
& \left(\frac{\partial \varphi}{\partial x_{i}}-\Pi^{\nabla} \frac{\partial \varphi}{\partial x_{i}}, \mathbf{b}_{i} v_{h}\right)_{E}+\left(\mathbf{b}_{i} \Pi^{\nabla} \frac{\partial \varphi}{\partial x_{i}}, v_{h}-\Pi^{0} v_{h}\right)_{E} \\
= & \left(\frac{\partial \varphi}{\partial x_{i}}-\Pi^{\nabla} \frac{\partial \varphi}{\partial x_{i}}, \mathbf{b}_{i} v_{h}-\Pi^{\nabla}\left(\mathbf{b}_{i} v_{h}\right)\right)_{E}+\left(\mathbf{b}_{i} \Pi^{\nabla} \frac{\partial \varphi}{\partial x_{i}}-\Pi^{\nabla}\left(\mathbf{b}_{i} \Pi^{\nabla} \frac{\partial \varphi}{\partial x_{i}}\right), v_{h}-\Pi^{0} v_{h}\right)_{E} \\
\leq & \left\|\frac{\partial \varphi}{\partial x_{i}}-\Pi^{\nabla} \frac{\partial \varphi}{\partial x_{i}}\right\|_{E}\left\|\mathbf{b}_{i} v_{h}-\Pi^{\nabla}\left(\mathbf{b}_{i} v_{h}\right)\right\|_{E}+\left\|\mathbf{b}_{i} \Pi^{\nabla} \frac{\partial \varphi}{\partial x_{i}}-\Pi^{\nabla}\left(\mathbf{b}_{i} \Pi^{\nabla} \frac{\partial \varphi}{\partial x_{i}}\right)\right\|_{E}\left\|v_{h}-\Pi^{0} v_{h}\right\|_{E} \\
\leq & C h_{E}\left(h_{E}|\varphi|_{2, E} \cdot\left|\mathbf{b}_{i} v_{h}\right|_{1, E}+\left\|\mathbf{b}_{i} \Pi^{\nabla} \frac{\partial \varphi}{\partial x_{i}}-\Pi^{\nabla}\left(\mathbf{b}_{i} \Pi^{\nabla} \frac{\partial \varphi}{\partial x_{i}}\right)\right\|_{E}\left\|v_{h}\right\|_{1, E}\right) \\
\leq & C h_{E}\left(\left\|\mathbf{b}_{i}\right\|_{W_{\infty}^{1}(E)} h_{E}|\varphi|_{2, E}\left|v_{h}\right|_{1, E}+\left\|\mathbf{b}_{i} \Pi^{\nabla} \frac{\partial \varphi}{\partial x_{i}}-\Pi^{\nabla}\left(\mathbf{b}_{i} \Pi^{\nabla} \frac{\partial \varphi}{\partial x_{i}}\right)\right\|_{E}\left\|v_{h}\right\|_{1, E}\right) .
\end{aligned}
$$

Taking into account the inequality (3.1), we obtain

$$
\left\|\mathbf{b}_{i} \Pi^{\nabla} \frac{\partial \varphi}{\partial x_{i}}-\Pi^{\nabla}\left(\mathbf{b}_{i} \Pi^{\nabla} \frac{\partial \varphi}{\partial x_{i}}\right)\right\|_{E} \leq C\left\|\mathbf{b}_{i} \Pi^{\nabla} \frac{\partial \varphi}{\partial x_{i}}-\Pi^{\nabla}\left(\mathbf{b}_{i} \frac{\partial \varphi}{\partial x_{i}}\right)\right\|_{E}
$$




$$
\begin{aligned}
& \leq C\left(\left\|\mathbf{b}_{i} \Pi^{\nabla} \frac{\partial \varphi}{\partial x_{i}}-\mathbf{b}_{i} \frac{\partial \varphi}{\partial x_{i}}\right\|_{E}+\left\|\mathbf{b}_{i} \frac{\partial \varphi}{\partial x_{i}}-\Pi^{\nabla}\left(\mathbf{b}_{i} \frac{\partial \varphi}{\partial x_{i}}\right)\right\|_{E}\right) \\
& \leq C\left\|\mathbf{b}_{i}\right\|_{W_{\infty}^{1}(E)} h_{E}|\varphi|_{2, E},
\end{aligned}
$$

which yields the estimate (3.6).

Lemma 3.4. If $E \in \mathscr{T}_{h}, K_{E} \ll h_{E}$ and $\varphi$ is a sufficiently regular function, then

$$
d_{h}\left(\varphi, v_{h}\right) \leq C \max _{E \in \mathscr{T}_{h}} \frac{K_{E}}{K_{E}^{V}}\|\sqrt{K} \nabla \varphi\|\left\|\sqrt{K} \nabla v_{h}\right\| .
$$

Moreover, if $K \in W_{\infty}^{1}(\Omega)$ and $\mathbf{b} \in\left[W_{\infty}^{1}(\Omega)\right]^{2}$, then

$$
\left|d\left(\varphi, v_{h}\right)-d_{h}\left(\varphi, v_{h}\right)\right| \leq C \max _{E \in \mathscr{T}_{h}} \frac{\|\mathbf{b}\|_{W_{\infty}^{1}(E)}\|\mathbf{K}\|_{W_{\infty}^{1}(E)} K_{E}}{\sqrt{K_{E}^{V}}} h\|\varphi\|_{2}\left\|\sqrt{K} \nabla v_{h}\right\| .
$$

Proof. If $\nabla \cdot(K \nabla \varphi)=0$, the inequality (3.8) is trivial, so we assume that $\nabla \cdot(K \nabla \varphi) \neq 0$. It follows from (2.2), the Cauchy-Schwarz inequality and the continuity of the projection $\Pi^{\nabla}$ that

$$
\begin{aligned}
d_{h}^{E}\left(\varphi, v_{h}\right)= & \tau_{E}\left(\left(\mathbf{b} \cdot \nabla\left(\Pi^{\nabla} \varphi\right), \nabla \cdot\left(K \nabla\left(\Pi^{\nabla} v_{h}\right)\right)\right)_{E}\right. \\
& \left.-\left(\nabla \cdot\left(K \nabla\left(\Pi^{\nabla} \varphi\right)\right), \mathbf{b} \cdot \nabla\left(\Pi^{\nabla} v_{h}\right)\right)_{E}\right) \\
\leq & \tau_{E}\left(\left\|\mathbf{b} \cdot \nabla\left(\Pi^{\nabla} \varphi\right)\right\|_{E}\left\|\nabla \cdot\left(K \nabla\left(\Pi^{\nabla} v_{h}\right)\right)\right\|_{E}\right. \\
& \left.+\left\|\nabla \cdot\left(K \nabla \Pi^{\nabla} \varphi\right)\right\|_{E}\left\|\mathbf{b} \cdot \nabla\left(\Pi^{\nabla} v_{h}\right)\right\|_{E}\right) \\
\leq & C \frac{\tau_{E} K_{E} b_{E}}{h_{E}}\left\|\nabla\left(\Pi^{\nabla} \varphi\right)\right\|_{E}\left\|\nabla\left(\Pi^{\nabla} v_{h}\right)\right\|_{E} \\
\leq & C \frac{K_{E}}{K_{E}^{V}}\|\sqrt{K} \nabla \varphi\|_{E}\left\|\sqrt{K} \nabla v_{h}\right\|_{E},
\end{aligned}
$$

and (3.8) is easily obtained.

We now show the inequality (3.9). Indeed, we have

$$
\begin{aligned}
& \left|d^{E}\left(\varphi, v_{h}\right)-d_{h}^{E}\left(\varphi, v_{h}\right)\right|=\tau_{E} \mid\left(\mathbf{b} \cdot \nabla \varphi, \nabla \cdot\left(K \nabla v_{h}\right)\right)-\left(\nabla \cdot(K \nabla \varphi), \mathbf{b} \cdot \nabla v_{h}\right) \\
& -\left.\left(\left(\mathbf{b} \cdot \nabla\left(\Pi^{\nabla} \varphi\right), \nabla \cdot\left(K \nabla\left(\Pi^{\nabla} v_{h}\right)\right)\right)-\left(\nabla \cdot\left(K \nabla\left(\Pi^{\nabla} \varphi\right)\right), \mathbf{b} \cdot \nabla\left(\Pi^{\nabla} v_{h}\right)\right)\right)\right|_{E} \\
\leq & \tau_{E}\left|\left(\nabla \cdot(K \nabla \varphi), \mathbf{b} \cdot \nabla v_{h}\right)_{E}-\left(\nabla \cdot\left(K \nabla\left(\Pi^{\nabla} \varphi\right)\right), \mathbf{b} \cdot \nabla\left(\Pi^{\nabla} v_{h}\right)\right)_{E}\right| \\
& +\tau_{E}\left|\left(\mathbf{b} \cdot \nabla \varphi, \nabla \cdot\left(K \nabla v_{h}\right)\right)-\left(\mathbf{b} \cdot \nabla\left(\Pi^{\nabla} \varphi\right), \nabla \cdot\left(K \nabla\left(\Pi^{\nabla} v_{h}\right)\right)\right)\right|_{E} .
\end{aligned}
$$

Both moduli in the right hand-side of (3.10) are estimated analogously, so we only consider the first one - i.e.

$$
\tau_{E}\left|\left(\nabla \cdot(K \nabla \varphi), \mathbf{b} \cdot \nabla v_{h}\right)_{E}-\left(\nabla \cdot\left(K \nabla\left(\Pi^{\nabla} \varphi\right)\right), \mathbf{b} \cdot \nabla\left(\Pi^{\nabla} v_{h}\right)\right)_{E}\right|
$$




$$
\begin{aligned}
& \leq \tau_{E}\left|\left(\nabla \cdot\left(K \nabla \varphi-K \nabla\left(\Pi^{\nabla} \varphi\right)\right), \mathbf{b} \cdot \nabla v_{h}\right)_{E}\right| \\
& \quad+\tau_{E}\left|\left(\nabla \cdot\left(K \nabla\left(\Pi^{\nabla} \varphi\right)\right), \mathbf{b} \cdot\left(\nabla v_{h}-\nabla\left(\Pi^{\nabla} v_{h}\right)\right)\right)_{E}\right| .
\end{aligned}
$$

Assuming that $\nabla \cdot\left(K \nabla \varphi-K \nabla\left(\Pi^{\nabla} \varphi\right)\right) \neq 0$, we employ the Cauchy-Schwarz inequality, (3.1) and (2.2) to obtain

$$
\begin{aligned}
& \tau_{E}\left|\left(\nabla \cdot\left(K \nabla \varphi-K \nabla\left(\Pi^{\nabla} \varphi\right)\right), \mathbf{b} \cdot \nabla v_{h}\right)_{E}\right| \leq \tau_{E}\left\|\nabla \cdot\left(K \nabla \varphi-K \nabla\left(\Pi^{\nabla} \varphi\right)\right)\right\|_{E}\left\|\mathbf{b} \cdot \nabla v_{h}\right\|_{E} \\
\leq & \frac{\tau_{E} b_{E}}{\sqrt{C_{E}} h_{E}}\left\|K \nabla \varphi-K \nabla\left(\Pi^{\nabla} \varphi\right)\right\|_{E}\left\|\nabla v_{h}\right\|_{E} \leq \frac{\|K\|_{W_{\infty}^{1}} b_{E}}{8 K_{E} \sqrt{C_{E}}} h_{E}\left\|\nabla \varphi-\nabla\left(\Pi^{\nabla} \varphi\right)\right\|_{E}\left\|\nabla v_{h}\right\|_{E} \\
\leq & C \frac{b_{E}}{\sqrt{C_{E}} \sqrt{K_{E}^{V}}} h_{E}^{2}|\varphi|_{2, E}\left\|\sqrt{K} \nabla v_{h}\right\|_{E} .
\end{aligned}
$$

Considering the second term in the right-hand side of (3.11), we have

$$
\begin{aligned}
& \tau_{E}\left(\nabla \cdot\left(K \nabla\left(\Pi^{\nabla} \varphi\right)\right), \mathbf{b} \cdot\left(\nabla v_{h}-\nabla\left(\Pi^{\nabla} v_{h}\right)\right)\right)_{E} \\
= & \tau_{E} \sum_{i=1}^{2}\left(\mathbf{b}_{i} \nabla \cdot\left(K \nabla\left(\Pi^{\nabla} \varphi\right)\right), \frac{\partial v_{h}}{\partial x_{i}}-\Pi^{\nabla}\left(\frac{\partial v_{h}}{\partial x_{i}}\right)\right)_{E},
\end{aligned}
$$

and the summands of (3.13) are estimated by the Cauchy-Schwarz inequality — viz.

$$
\begin{aligned}
& \tau_{E}\left(\mathbf{b}_{i} \nabla \cdot\left(K \nabla\left(\Pi^{\nabla} \varphi\right)\right), \frac{\partial v_{h}}{\partial x_{i}}-\Pi^{\nabla}\left(\frac{\partial v_{h}}{\partial x_{i}}\right)\right)_{E} \\
= & \tau_{E}\left(\nabla \cdot\left(\mathbf{b}_{i} K \nabla\left(\Pi^{\nabla} \varphi\right)\right), \frac{\partial v_{h}}{\partial x_{i}}-\Pi^{\nabla}\left(\frac{\partial v_{h}}{\partial x_{i}}\right)\right)_{E} \\
& -\tau_{E}\left(\nabla \cdot \mathbf{b}_{i}\left(K \nabla\left(\Pi^{\nabla} \varphi\right)\right), \frac{\partial v_{h}}{\partial x_{i}}-\Pi^{\nabla}\left(\frac{\partial v_{h}}{\partial x_{i}}\right)\right)_{E} \\
= & \tau_{E}\left(\nabla \cdot\left(\mathbf{b}_{i} K \nabla\left(\Pi^{\nabla} \varphi\right)\right)-\nabla \cdot\left(\Pi^{\nabla}\left(\mathbf{b}_{i}\left(K \nabla\left(\Pi^{\nabla} \varphi\right)\right)\right)\right), \frac{\partial v_{h}}{\partial x_{i}}-\Pi^{\nabla}\left(\frac{\partial v_{h}}{\partial x_{i}}\right)\right)_{E} \\
& +\tau_{E}\left(\Pi^{\nabla}\left(\nabla \mathbf{b}_{i} \cdot\left(K \nabla\left(\Pi^{\nabla} \varphi\right)\right)\right)-\nabla \mathbf{b}_{i}\left(K \nabla\left(\Pi^{\nabla} \varphi\right)\right), \frac{\partial v_{h}}{\partial x_{i}}-\Pi^{\nabla}\left(\frac{\partial v_{h}}{\partial x_{i}}\right)\right)_{E} \\
\leq & \tau_{E}\left\|\frac{\partial v_{h}}{\partial x_{i}}-\Pi^{\nabla}\left(\frac{\partial v_{h}}{\partial x_{i}}\right)\right\|_{E}\left(\left\|\nabla \cdot\left(\mathbf{b}_{i} K \nabla\left(\Pi^{\nabla} \varphi\right)\right)-\nabla \cdot\left(\Pi^{\nabla}\left(\mathbf{b}_{i}\left(K \nabla\left(\Pi^{\nabla} \varphi\right)\right)\right)\right)\right\|_{E}\right. \\
& \left.+\left\|\Pi^{\nabla}\left(\nabla \mathbf{b}_{i} \cdot\left(K \nabla\left(\Pi^{\nabla} \varphi\right)\right)\right)-\nabla \mathbf{b}_{i}\left(K \nabla\left(\Pi^{\nabla} \varphi\right)\right)\right\|_{E}\right) \\
\leq & \frac{\tau_{E}}{\sqrt{K_{E}^{V}}}\left\|\nabla v_{h}\right\|_{E}\left(\left\|\nabla \cdot\left(\mathbf{b}_{i} K \nabla\left(\Pi^{\nabla} \varphi\right)\right)-\Pi^{\nabla}\left(\mathbf{b}_{i}\left(K \nabla\left(\Pi^{\nabla} \varphi\right)\right)\right)\right\|_{E}\right. \\
& \left.+\left\|\Pi^{\nabla}\left(\sqrt{K} \nabla \mathbf{b}_{i} \cdot\left(K \nabla\left(\Pi^{\nabla} \varphi\right)\right)\right)-\nabla \mathbf{b}_{i}\left(K \nabla\left(\Pi^{\nabla} \varphi\right)\right)\right\|_{E}\right) .
\end{aligned}
$$

Since $\Pi^{\nabla}$ is the best $L^{2}(E)$-approximation in $\mathbb{P}(E)$, we can use the inequalities (3.1),(2.2) to obtain

$$
\tau_{E}\left\|\nabla \cdot\left(\mathbf{b}_{i} K \nabla\left(\Pi^{\nabla} \varphi\right)\right)-\Pi^{\nabla}\left(\mathbf{b}_{i}\left(K \nabla\left(\Pi^{\nabla} \varphi\right)\right)\right)\right\|_{E}
$$




$$
\begin{aligned}
& \leq \frac{C_{E} h_{E}^{2}}{K_{E}}\left\|\nabla \cdot\left(\mathbf{b}_{i} K \nabla\left(\Pi^{\nabla} \varphi\right)\right)-\Pi^{\nabla}\left(\mathbf{b}_{i}\left(K \nabla\left(\Pi^{\nabla} \varphi\right)\right)\right)\right\|_{E} \\
& \leq \frac{\sqrt{C_{E}} h_{E}}{K_{E}}\left\|\mathbf{b}_{i} K \nabla\left(\Pi^{\nabla} \varphi\right)-\Pi^{\nabla}\left(\mathbf{b}_{i}\left(K \nabla\left(\Pi^{\nabla} \varphi\right)\right)\right)\right\|_{E} \\
& \leq \frac{\sqrt{C_{E}} h_{E}}{K_{E}}\left\|\mathbf{b}_{i} K \nabla\left(\Pi^{\nabla} \varphi\right)-\Pi^{\nabla}\left(\mathbf{b}_{i}(K \nabla \varphi)\right)\right\|_{E} \\
& \leq \frac{\sqrt{C_{E}} h_{E}}{K_{E}}\left(\left\|\mathbf{b}_{i} K \nabla\left(\Pi^{\nabla} \varphi-\varphi\right)\right\|_{E}+\left\|\mathbf{b}_{i} K \nabla \varphi-\Pi^{\nabla}\left(\mathbf{b}_{i}(K \nabla \varphi)\right)\right\|_{E}\right) \\
& \leq C \frac{\sqrt{C_{E}} h_{E}}{K_{E}}\left(h_{E} b_{E} K_{E}|\varphi|_{2, E}+h_{E}\left|\mathbf{b}_{i} K \nabla \varphi\right|_{1, E}\right) \\
& \leq C \sqrt{C_{E}} b_{E} h_{E}^{2}\left(|\varphi|_{2, E}+\|\varphi\|_{2, E}\right)
\end{aligned}
$$

and

$$
\begin{aligned}
& \tau_{E}\left\|\Pi^{\nabla}\left(\nabla \mathbf{b}_{i} \cdot\left(K \nabla\left(\Pi^{\nabla} \varphi\right)\right)\right)-\nabla \mathbf{b}_{i}\left(K \nabla\left(\Pi^{\nabla} \varphi\right)\right)\right\|_{E} \\
\leq & \tau_{E}\left\|\Pi^{\nabla}\left(\nabla \mathbf{b}_{i} \cdot(K \nabla \varphi)\right)-\nabla \mathbf{b}_{i}\left(K \nabla\left(\Pi^{\nabla} \varphi\right)\right)\right\|_{E} \\
\leq & \frac{h_{E}}{2 b_{E}}\left(\left\|\Pi^{\nabla}\left(\nabla \mathbf{b}_{i} \cdot(K \nabla \varphi)\right)-\nabla \mathbf{b}_{i} K \nabla \varphi\right\|+\left\|\nabla \mathbf{b}_{i} K \nabla \varphi-\nabla \mathbf{b}_{i}\left(K \nabla\left(\Pi^{\nabla} \varphi\right)\right)\right\|_{1, E}\right) \\
\leq & \frac{h_{E}}{2 b_{E}}\left(h_{E}\left|\nabla \mathbf{b}_{i} K \nabla \varphi\right|_{1, E}+h_{E} K_{E} b_{E}|\varphi|_{2, E}\right) \leq \frac{K_{E} h_{E}^{2}}{2}\left(\|\varphi\|_{2, E}+|\varphi|_{2, E}\right) .
\end{aligned}
$$

Combining estimates in (3.11)-(3.16) leads to the inequality

$$
\begin{aligned}
& \left|\sum_{E \in \mathscr{T}_{h}}\left(\tau_{E}\left(\nabla \cdot(K \nabla \varphi), \mathbf{b} \cdot \nabla v_{h}\right)_{E}-\sum_{E \in \mathscr{T}_{h}} \tau_{E}\left(\nabla \cdot\left(K \nabla\left(\Pi^{\nabla} \varphi\right)\right), \mathbf{b} \cdot \nabla\left(\Pi^{\nabla} v_{h}\right)\right)\right)_{E}\right| \\
\leq & C \max _{E \in \mathscr{T}_{h}} \frac{\|\mathbf{b}\|_{W_{\infty}^{1}(E)}\|\mathbf{K}\|_{W_{\infty}^{1}(E)}\left(K_{E}+b_{E}\right)}{K_{E}^{V}} h^{2}\|\varphi\|_{2}\left\|\sqrt{K} \nabla v_{h}\right\| \quad \text { for any } \quad E \in \mathscr{T}_{h} .
\end{aligned}
$$

Analogous considerations show that

$$
\begin{aligned}
& \tau_{E}\left|\left(\mathbf{b} \cdot \nabla \varphi, \nabla \cdot\left(K \nabla v_{h}\right)\right)-\left(\mathbf{b} \cdot \nabla\left(\Pi^{\nabla} \varphi\right), \nabla \cdot\left(K \nabla\left(\Pi^{\nabla} v_{h}\right)\right)\right)\right|_{E} \\
\leq & C \max _{E \in \mathscr{T}_{h}} \frac{\|\mathbf{b}\|_{W_{\infty}^{1}(E)} K_{E}}{\sqrt{K_{E}^{V}}} h\|\varphi\|_{2}\left\|\nabla v_{h}\right\| \quad \text { for any } \quad E \in \mathscr{T}_{h},
\end{aligned}
$$

and estimate (3.9) follows from (3.10),(3.17) and (3.18).

\subsection{Well-posedness of discrete problem}

We now show that the problem (2.5) is well-posed. 
Lemma 3.5. There exists a constant $\alpha>0$ such that for any $v_{h} \in V_{h}(\Omega)$ the inequality

$$
a_{h}\left(v_{h}, v_{h}\right) \geq \alpha\left\|\nabla v_{h}\right\|^{2}
$$

holds.

Proof. If $v_{h} \in V_{h}(\Omega)$ and $E \in \mathscr{T}_{h}$, then

$$
\begin{aligned}
a_{h}^{E}\left(v_{h}, v_{h}\right)= & \left\|\sqrt{K} \nabla\left(\Pi^{\nabla} v_{h}\right)\right\|_{E}^{2}+\tau_{E}\left\|\mathbf{b} \cdot \nabla\left(\Pi^{\nabla} v_{h}\right)\right\|_{E}^{2}-\tau_{E}\left\|\nabla \cdot\left(K \nabla\left(\Pi^{\nabla} v_{h}\right)\right)\right\|_{E}^{2} \\
& +\left(K_{E}+\tau_{E} b_{E}^{2}\right) S_{a}^{E}\left(\left(I-\Pi^{\nabla}\right) v_{h},\left(I-\Pi^{\nabla}\right) v_{h}\right) .
\end{aligned}
$$

The relation (2.3) and the properties of the orthogonal projection show that there is $c^{*}>0$ such that for any $E \in \mathscr{T}_{h}$ the inequality

$$
S_{a}^{E}\left(\left(I-\Pi^{\nabla}\right) v_{h},\left(I-\Pi^{\nabla}\right) v_{h}\right) \geq c^{*}\left\|\nabla\left(v_{h}-\Pi^{\nabla} v_{h}\right)\right\|_{E}^{2}
$$

holds. It follows from the definition of $\tau_{E}$ and the inverse inequality that

$$
\tau_{E}\left\|\nabla \cdot\left(K \nabla\left(\Pi^{\nabla} v_{h}\right)\right)\right\|_{E}^{2} \leq \frac{C K_{E}}{b_{E} h_{E}}\left\|\sqrt{K_{E}} \nabla\left(\Pi^{\nabla} v_{h}\right)\right\|_{E}^{2} .
$$

Setting

$$
\alpha=\min _{E \in \mathscr{T}_{h}}\left\{\min \left\{c^{*}, 1\right\}-\frac{C K_{E}}{b_{E} h_{E}}\right\}
$$

we obtain

$$
\begin{aligned}
a_{h}^{E}\left(v_{h}, v_{h}\right) \geq & \left\|\sqrt{K} \nabla\left(\Pi^{\nabla} v_{h}\right)\right\|_{E}^{2}+\tau_{E}\left\|\mathbf{b} \cdot \nabla\left(\Pi^{\nabla} v_{h}\right)\right\|_{E}^{2}-\tau_{E}\left\|\nabla \cdot\left(K \nabla\left(\Pi^{\nabla} v_{h}\right)\right)\right\|_{E}^{2} \\
& +c^{*}\left(\left\|\sqrt{K}\left(\nabla v_{h}-\Pi^{\nabla} \nabla v_{h}\right)\right\|_{E}^{2}+\tau_{E}\left\|\mathbf{b} \cdot\left(\nabla v_{h}-\Pi^{\nabla} \nabla v_{h}\right)\right\|_{E}^{2}\right) \\
\geq & \min \left\{c^{*}, 1\right\}\left(\left\|\sqrt{K} \nabla\left(\Pi^{\nabla} v_{h}\right)\right\|_{E}^{2}+\left\|\sqrt{K}\left(\nabla v_{h}-\Pi^{\nabla} \nabla v_{h}\right)\right\|_{E}^{2}\right. \\
& \left.+\tau_{E}\left\|\mathbf{b} \cdot \nabla\left(\Pi^{\nabla} v_{h}\right)\right\|_{E}^{2}+\tau_{E}\left\|\mathbf{b} \cdot\left(\nabla v_{h}-\Pi^{\nabla} \nabla v_{h}\right)\right\|_{E}^{2}\right) \\
& -\frac{C K_{E}}{b_{E} h_{E}}\left\|\sqrt{K_{E}} \nabla\left(\Pi^{\nabla} v_{h}\right)\right\|_{E}^{2} \\
\geq & \left(\min \left\{c^{*}, 1\right\}-\frac{C K_{E}}{b_{E} h_{E}}\right)\left(\left\|\sqrt{K} \nabla v_{h}\right\|_{E}^{2}+\tau_{E}\left\|\mathbf{b} \cdot \nabla v_{h}\right\|_{E}^{2}\right)
\end{aligned}
$$

which completes the proof.

Remark 3.1. If $E \in \mathscr{T}_{h}$, then $K_{E} \ll h_{E}$, and one has

$$
\alpha=: \min _{E \in \mathscr{T}_{h}}\left\{\min \left\{c^{*}, 1\right\}-\frac{C K_{E}}{b_{E} h_{E}}\right\}>0 .
$$

We now consider the set of functions $v_{h} \in H_{0}^{1}(\Omega)$ and equip it with the norm

$$
\left\|v_{h}\right\|:=\left\{\left\|\sqrt{K} \nabla v_{h}\right\|^{2}+\sum_{E \in \mathscr{T}_{h}} \tau_{E}\left\|\mathbf{b} \cdot \nabla v_{h}\right\|_{E}^{2}\right\}^{1 / 2} .
$$


Lemma 3.6. If $q \in H_{0}^{1}(\Omega)$, then there is $q_{h} \in V_{h}(\Omega)$ such that

$$
a_{h}\left(q_{h}, v_{h}\right)=a\left(q, v_{h}\right) \text { for any } v_{h} \in V_{h}(\Omega) .
$$

Moreover,

$$
\begin{aligned}
& \left\|q_{h}\right\| \leq \frac{1}{\alpha}\|q\|, \\
& \left\|q-q_{h}\right\| \leq C h\|q\|,
\end{aligned}
$$

where $\alpha$ is the coercivity constant in (3.19).

Proof. The proof follows the pattern of the proof of [5, Lemma 5.6)].

Lemma 3.7. For any $v_{h} \in V_{h}(\Omega)$ the inequality

$$
A_{s}\left(v_{h}, v_{h}\right) \geq \frac{7}{8}\left\|v_{h}\right\|^{2}
$$

holds.

Proof. The homogeneous Dirichlet boundary conditions and the equation $\nabla \cdot \mathbf{b}=0$ yield

$$
\left(\mathbf{b} \cdot \nabla v_{h}, v_{h}\right)=-\frac{1}{2}\left(\nabla \cdot \mathbf{b}, v_{h}^{2}\right)=0, \quad \forall v_{h} \in V_{h}(\Omega) .
$$

Therefore, one can use the estimate (2.2) and the Cauchy-Schwarz and Young inequalities to obtain

$$
\begin{aligned}
A_{s}\left(v_{h}, v_{h}\right) & =\left\|\sqrt{K} \nabla v_{h}\right\|^{2}+\sum_{E \in \mathscr{T}_{h}} \tau_{E}\left\|\mathbf{b} \cdot \nabla v_{h}\right\|_{E}^{2}-\sum_{E \in \mathscr{T}_{h}} \tau_{E}\left(\nabla \cdot\left(K \nabla v_{h}\right), \nabla \cdot\left(K \nabla v_{h}\right)\right)_{E} \\
& \geq\left\|\sqrt{K} \nabla v_{h}\right\|^{2}+\sum_{E \in \mathscr{T}_{h}} \tau_{E}\left\|\mathbf{b} \cdot \nabla v_{h}\right\|_{E}^{2}-\sum_{E \in \mathscr{T}_{h}} \tau_{E}\left\|\nabla \cdot\left(K \nabla v_{h}\right)\right\|_{E}^{2} \\
& \geq\left\|\sqrt{K} \nabla v_{h}\right\|^{2}+\sum_{E \in \mathscr{T}_{h}} \tau_{E}\left\|\mathbf{b} \cdot \nabla v_{h}\right\|_{E}^{2}-\sum_{E \in \mathscr{T}_{h}} \frac{1}{8}\left\|\sqrt{K} \nabla v_{h}\right\|_{E}^{2} \\
& \geq \frac{7}{8}\left\|\sqrt{K} \nabla v_{h}\right\|^{2}+\sum_{E \in \mathscr{T}_{h}} \tau_{E}\left\|\mathbf{b} \cdot \nabla v_{h}\right\|_{E}^{2} \\
& \geq \frac{7}{8}\left(\left\|\sqrt{K} \nabla v_{h}\right\|^{2}+\sum_{E \in \mathscr{T}_{h}} \tau_{E}\left\|\mathbf{b} \cdot \nabla v_{h}\right\|_{E}^{2}\right) \geq \frac{7}{8}\left\|v_{h}\right\|^{2}
\end{aligned}
$$

as stated.

Theorem 3.1. If $K \in L^{\infty}(\Omega)$ and $\mathbf{b} \in\left[W_{\infty}^{1}(\Omega)\right]^{2}$, then for any sufficiently small $h$ and any $v_{h} \in V_{h}(\Omega)$, the inequality

$$
\sup _{w_{h} \in V_{h}} \frac{A_{s, h}\left(v_{h}, w_{h}\right)}{\left\|w_{h}\right\|} \geq C\left\|\mid v_{h}\right\|
$$

holds. 
Proof. Assume that for any $w_{h} \in V_{h}(\Omega)$, the elements $v_{h} \in V_{h}(\Omega)$ and $v_{h}^{*} \in V_{h}(\Omega)$ satisfy the equation

$$
a_{h}\left(v_{h}^{*}, w_{h}\right)=a\left(v_{h}, w_{h}\right)
$$

The symmetry of $a_{h}$ implies that

$$
\begin{aligned}
A_{s, h}\left(v_{h}, v_{h}^{*}\right) & =a_{h}\left(v_{h}, v_{h}^{*}\right)+b_{h}\left(v_{h}, v_{h}^{*}\right)+d_{h}\left(v_{h}, v_{h}^{*}\right) \\
& =a\left(v_{h}, v_{h}\right)+b_{h}\left(v_{h}, v_{h}^{*}\right)+d_{h}\left(v_{h}, v_{h}^{*}\right) \\
& =A_{s}\left(v_{h}, v_{h}^{*}\right)+r\left(v_{h}, v_{h}^{*}\right),
\end{aligned}
$$

where

$$
r\left(v_{h}, v_{h}^{*}\right)=b_{h}\left(v_{h}, v_{h}^{*}\right)-b\left(v_{h}, v_{h}^{*}\right)+b\left(v_{h}, v_{h}^{*}-v_{h}\right)+d_{h}\left(v_{h}, v_{h}^{*}\right)-d\left(v_{h}, v_{h}^{*}\right)+d\left(v_{h}, v_{h}^{*}-v_{h}\right) .
$$

By Lemmas 3.3 and 3.4, the forms $b$ and $d$ are continuous and it follows from (3.20), (3.21) that

$$
\left|r\left(v_{h}, v_{h}^{*}\right)\right| \leq C_{r} h\left\|\sqrt{K} \nabla v_{h}\right\|\left\|\sqrt{K} \nabla v_{h}^{*}\right\| \leq C_{r} h\left|\left\|v _ { h } \left|\left\|\left|\left\|v_{h}^{*}\right\|\right| \leq\left. C_{r} h\left|\| v_{h}\right|\right|^{2},\right.\right.\right.\right.
$$

where $C_{r}>0$ depends on $\|K\|_{L^{\infty}(\Omega)},\|\mathbf{b}\|_{W_{\infty}(\Omega)}^{1}$ and on the approximation constant in (3.1).

If $h \leq(7 \alpha) /\left(8 C_{r}\right)$, the inequalities (3.20) and (3.23) yield

$$
A_{s, h}\left(v_{h}, v_{h}\right) \geq \frac{7}{8}\left\|v_{h}\right\|^{2}+r\left(v_{h}, v_{h}^{*}\right) \geq\left(\frac{7}{8} \alpha-C_{r} h\right)\left\|\left|v_{h}\|\mid\| v_{h}^{*}\|\|\right.\right.
$$

and the estimate (3.22) follows.

\subsection{A priori error estimate}

Theorem 3.2. If $u \in H^{2}(\Omega), K \in W_{\infty}^{1}(\Omega)$ and $\mathbf{b} \in\left[W_{\infty}^{2}(\Omega)\right]^{2}$, then for all sufficiently small $h$, one has

$$
\left\|u-u_{h}\right\| \mid \leq C h\left(\|u\|_{2}+\|f\|_{1}\right) .
$$

Proof. Considering a VEM interpolator $u_{I}$ of $u$, we write

$$
\left\|u-u_{h}\right\|^{2} \leq\left\|u-u_{I}\right\|^{2}+\left\|u_{h}-u_{I}\right\|^{2} .
$$

By [5, Lemma 5.1], for any $E \in \mathscr{T}_{h}$ and any $w \in H^{2}$, the inequality

$$
\left\|w-w_{h}\right\|_{1, E} \leq h_{E}|w|_{2, E}
$$

holds. Therefore,

$$
\begin{aligned}
\left\|u-u_{I}\right\|^{2} & =\sum_{E \in \mathscr{T}_{h}}\left(\left\|\sqrt{K} \nabla\left(u-u_{I}\right)\right\|_{E}^{2}+\tau_{E}\left\|\mathbf{b} \cdot \nabla\left(u-u_{I}\right)\right\|_{E}^{2}\right) \\
& \leq \sum_{E \in \mathscr{T}_{h}}\left(K_{E}+\tau_{E} b_{E}^{2}\right)\left\|\nabla\left(u-u_{I}\right)\right\|_{E}^{2} \leq \sum_{E \in \mathscr{T}_{h}}\left(K_{E}+\tau_{E} b_{E}^{2}\right) h_{E}^{2}|u|_{2, E}^{2} .
\end{aligned}
$$


Let us first estimate the element $e_{h}:=u_{h}-u_{I} \in V_{h}(\Omega)$. By Theorem 3.1, there is $w_{h} \in V_{h}(\Omega)$ such that

$$
\begin{aligned}
C\left\|\left|e _ { h } \left\|\left|\left\|w_{h}\right\|\right|\right.\right.\right. & \leq A_{s, h}\left(u_{h}-u_{I}, w_{h}\right)=L_{s, h}\left(w_{h}\right)-A_{s, h}\left(u_{I}, w_{h}\right) \\
& =L_{s, h}\left(w_{h}\right)-L_{s}\left(w_{h}\right)+A_{s}\left(u, w_{h}\right)-A_{s, h}\left(u_{I}, w_{h}\right) \\
& =L_{s, h}\left(w_{h}\right)-L_{s}\left(w_{h}\right)+A_{s, h}\left(u-u_{I}, w_{h}\right)+A_{s}\left(u, w_{h}\right)-A_{s, h}\left(u, w_{h}\right) .
\end{aligned}
$$

The first difference in the right-hand side of (3.26) is estimated as follows:

$$
\begin{aligned}
& L_{s, h}\left(w_{h}\right)-L_{s}\left(w_{h}\right)=\sum_{E \in \mathscr{T}_{h}}\left(f,\left(\Pi^{\nabla}-I\right) w_{h}+\mathbf{b} \cdot \nabla\left(\Pi^{\nabla}-I\right) w_{h}+\nabla \cdot\left(K \Pi^{\nabla}-I\right) w_{h}\right)_{E} \\
= & \sum_{E \in \mathscr{T}_{h}}\left(\left(f,\left(\Pi^{\nabla}-I\right) w_{h}\right)_{E}+\left(f, \mathbf{b} \cdot \nabla\left(\Pi^{\nabla}-I\right) w_{h}\right)_{E}+\left(f, \nabla \cdot K\left(\Pi^{\nabla}-I\right) w_{h}\right)_{E}\right) \\
= & \sum_{E \in \mathscr{T}_{h}}\left(\left(\left(I-\Pi^{\nabla}\right) f,\left(\Pi^{\nabla}-I\right) w_{h}\right)_{E}+\sum_{i=1}^{2}\left(\left(\Pi^{\nabla}-I\right)\left(\mathbf{b}_{i} f\right), \frac{\partial w_{h}}{\partial x_{i}}\right)_{E}\right. \\
& \left.+\left(\left(I-\Pi^{\nabla}\right) f, \nabla \cdot K\left(\Pi^{\nabla}-I\right) w_{h}\right)_{E}\right) \\
\leq & \sum_{E \in \mathscr{T}_{h}}\left(\left\|f-\Pi^{\nabla} f\right\|_{E}\left\|w_{h}-\Pi^{\nabla} w_{h}\right\|_{E}+\sum_{i=1}^{2}\left\|\left(\Pi^{\nabla}-I\right)\left(\mathbf{b}_{i} f\right)\right\|_{E}\left\|\frac{\partial w_{h}}{\partial x_{i}}\right\|_{E}\right. \\
& \left.+K_{E}\left\|f-\Pi^{\nabla} f\right\|_{E}\left\|\nabla\left(\Pi^{\nabla}-I\right) w_{h}\right\|_{E}\right) \\
\leq & \sum_{E \in \mathscr{T}_{h}} C\left(|f|_{1, E} h_{E}\left\|\nabla w_{h}\right\|_{E}+\frac{1}{\sqrt{K_{E}^{V}}} h_{E} \sum_{i=1}^{2}\left|\mathbf{b}_{i} f\right|_{1, E}\left\|\sqrt{K} \nabla w_{h}\right\|_{E}+K_{E} h_{E}|f|_{1, E}\left\|\nabla w_{h}\right\|_{E}\right) \\
\leq & \sum_{E \in \mathscr{T}_{h}} C\left(\frac{1}{\sqrt{K_{E}^{V}}} h_{E}\|f\|_{1, E}\left\|\sqrt{K} \nabla w_{h}\right\|_{E}+\frac{1}{\sqrt{K_{E}^{V}}} h_{E} \sum_{i=1}^{2}\left|\mathbf{b}_{i} f\right|_{1, E}\left\|\sqrt{K} \nabla w_{h}\right\|_{E}\right) \\
\leq & \sum_{E \in \mathscr{T}_{h}} C\left(\frac{1}{\sqrt{K_{E}^{V}}} h_{E}\|f\|_{1, E}\left|\left\|w_{h}\right\|\left\|_{E}+\frac{\|\mathbf{b}\|_{W_{\infty}^{1}(E)}^{1}}{\sqrt{K_{E}^{V}}} h_{E}\right\| f\left\|_{1, E} \mid\right\| w_{h}\|\|_{E}\right) .\right.
\end{aligned}
$$

Using the inequalities (3.3),(3.5),(3.8) and (3.25), we obtain

$$
A_{s, h}\left(u-u_{I}, w_{h}\right) \leq C\left\|u-u_{I}\right\|_{1}\left\|w_{h}\right\|_{1} \leq C h\|u\|_{2}\left\|w_{h}\right\| .
$$

The last difference in (3.26) is estimated by employing (3.4),(3.6) and (3.9), so that

$$
\begin{aligned}
\left|A_{h}\left(u, w_{h}\right)-A_{s, h}\left(u, w_{h}\right)\right| \leq & \left|a\left(u, w_{h}\right)-a_{h}\left(u, w_{h}\right)\right|+\left|b\left(u, w_{h}\right)-b_{h}\left(u, w_{h}\right)\right| \\
& +\left|d\left(u, w_{h}\right)-d_{h}\left(u, w_{h}\right)\right| \leq C h\|u\|_{2}\left|\| w_{h}\right| \mid,
\end{aligned}
$$

and (3.24) now follows from (3.26)-(3.29). 


\section{Numerical Experiments}

We consider approximate solutions of the problem (2.1) constructed by employing various meshes. In particular, Fig. 1 demonstrates the triangle, quadrangle and distorted quadrangle meshes in the domain $[0,1]^{2}$.

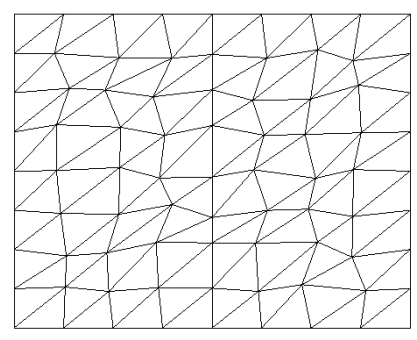

Mesh 1

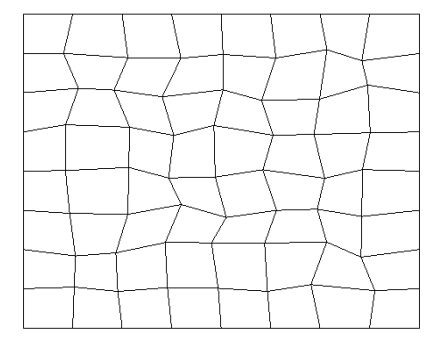

Mesh 2

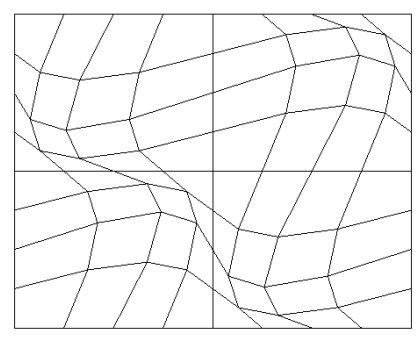

Mesh 3

Figure 1: Triangle, quadrangle and distorted quadrangle meshes (left to right).

Example 4.1. Consider the first example from [9], where the solution $u$ of the problem (2.1), the diffusion $K$ and the convection $\mathbf{b}$ are given by

$$
u=\frac{65536}{729} x^{3}(1-x) y^{3}(1-y), \quad K=v \mathbf{I}, \quad \mathbf{b}=\left(\frac{1}{2},-\frac{\sqrt{3}}{2}\right) .
$$

The right-hand side $f(\mathbf{x})$ is determined by substituting $u$ into the Eq. (2.1).

We carry out two sets of simulations for two different values of $K$ for $v=10^{-3}$ and $10^{-9}$. The errors corresponding to the degrees of freedom are shown in Tables 1-2. Note that the method maintains the optimal rate of convergence for both $L^{2}$ - and $H^{1}$ errors.

Table 1: Example 4.1: $v=10^{-3}$.

\begin{tabular}{||c|cc|cccccc||}
\hline Mesh & $D O F$ & Péclet & \|\|$u-u_{h} \|$ & order & $\left\|u-u_{h}\right\|_{0}$ & order & $\left\|u-u_{h}\right\|_{1}$ & order \\
\hline Mesh 1 & 81 & $1.08 \mathrm{e}+02$ & $2.6137 \mathrm{e}-01$ & - & $6.8355 \mathrm{e}-03$ & - & $2.6137 \mathrm{e}-01$ & - \\
& 289 & $5.41 \mathrm{e}+01$ & $1.2403 \mathrm{e}-01$ & 1.08 & $1.4332 \mathrm{e}-03$ & 2.25 & $1.2396 \mathrm{e}-01$ & 1.07 \\
& 1089 & $2.71 \mathrm{e}+01$ & $5.9553 \mathrm{e}-02$ & 1.06 & $3.2650 \mathrm{e}-04$ & 2.13 & $5.9523 \mathrm{e}-02$ & 1.06 \\
& 4225 & $1.36 \mathrm{e}+01$ & $2.9477 \mathrm{e}-02$ & 1.01 & $7.7903 \mathrm{e}-05$ & 2.06 & $2.9462 \mathrm{e}-02$ & 1.01 \\
& 16641 & $6.80 \mathrm{e}+00$ & $1.4703 \mathrm{e}-02$ & 1.00 & $1.9712 \mathrm{e}-05$ & 1.98 & $1.4693 \mathrm{e}-02$ & 1.00 \\
\hline Mesh 2 & 81 & $1.08 \mathrm{e}+02$ & $4.7368 \mathrm{e}-01$ & - & $2.6204 \mathrm{e}-02$ & - & $4.7344 \mathrm{e}-01$ & - \\
& 289 & $5.41 \mathrm{e}+01$ & $2.4801 \mathrm{e}-01$ & 0.93 & $4.8396 \mathrm{e}-03$ & 2.43 & $2.4788 \mathrm{e}-01$ & 0.93 \\
& 1089 & $2.71 \mathrm{e}+01$ & $1.2107 \mathrm{e}-01$ & 1.03 & $9.1985 \mathrm{e}-04$ & 2.40 & $1.2101 \mathrm{e}-01$ & 1.03 \\
& 4225 & $1.36 \mathrm{e}+01$ & $5.6082 \mathrm{e}-02$ & 1.11 & $2.0337 \mathrm{e}-04$ & 2.18 & $5.6054 \mathrm{e}-02$ & 1.11 \\
& 16641 & $6.80 \mathrm{e}+00$ & $2.6993 \mathrm{e}-02$ & 1.05 & $4.7849 \mathrm{e}-05$ & 2.08 & $2.6959 \mathrm{e}-02$ & 1.06 \\
\hline Mesh 3 & 81 & $1.08 \mathrm{e}+02$ & $6.7412 \mathrm{e}-01$ & - & $5.0924 \mathrm{e}-02$ & - & $6.7378 \mathrm{e}-01$ & - \\
& 289 & $5.41 \mathrm{e}+01$ & $2.8577 \mathrm{e}-01$ & 1.24 & $1.4581 \mathrm{e}-02$ & 1.80 & $2.8563 \mathrm{e}-01$ & 1.24 \\
& 1089 & $2.71 \mathrm{e}+01$ & $1.4148 \mathrm{e}-01$ & 1.01 & $3.6129 \mathrm{e}-03$ & 2.01 & $1.4141 \mathrm{e}-01$ & 1.01 \\
& 4225 & $1.36 \mathrm{e}+01$ & $6.9578 \mathrm{e}-02$ & 1.02 & $7.8590 \mathrm{e}-04$ & 2.20 & $6.9543 \mathrm{e}-02$ & 1.02 \\
& 16641 & $6.80 \mathrm{e}+00$ & $3.4568 \mathrm{e}-02$ & 1.01 & $1.7442 \mathrm{e}-04$ & 2.17 & $3.4585 \mathrm{e}-02$ & 1.01 \\
\hline
\end{tabular}


Table 2: Example 4.1: $v=10^{-9}$.

\begin{tabular}{||c|cc|cccccc||}
\hline Mesh & $D O F$ & Péclet & \|\|$u-u_{h} \|$ & order & $\left\|u-u_{h}\right\|_{0}$ & order & $\left\|u-u_{h}\right\|_{1}$ & order \\
\hline Mesh 1 & 81 & $1.08 \mathrm{e}+08$ & $2.6363 \mathrm{e}-01$ & - & $8.2778 \mathrm{e}-03$ & - & $2.6363 \mathrm{e}-01$ & - \\
& 289 & $5.41 \mathrm{e}+07$ & $1.2433 \mathrm{e}-01$ & 1.08 & $2.0521 \mathrm{e}-03$ & 2.01 & $1.2433 \mathrm{e}-01$ & 1.08 \\
& 1089 & $2.71 \mathrm{e}+07$ & $5.9720 \mathrm{e}-02$ & 1.06 & $4.8622 \mathrm{e}-04$ & 2.08 & $5.9720 \mathrm{e}-02$ & 1.06 \\
& 4225 & $1.36 \mathrm{e}+07$ & $2.9665 \mathrm{e}-02$ & 1.01 & $1.1432 \mathrm{e}-04$ & 2.09 & $2.9665 \mathrm{e}-02$ & 1.01 \\
& 16641 & $6.80 \mathrm{e}+06$ & $1.4105 \mathrm{e}-02$ & 1.07 & $2.8190 \mathrm{e}-05$ & 2.02 & $1.4105 \mathrm{e}-02$ & 1.07 \\
\hline Mesh 2 & 81 & $1.08 \mathrm{e}+08$ & $4.7481 \mathrm{e}-01$ & - & $2.6481 \mathrm{e}-02$ & - & $4.7481 \mathrm{e}-01$ & - \\
& 289 & $5.41 \mathrm{e}+07$ & $2.4843 \mathrm{e}-01$ & 0.93 & $4.9354 \mathrm{e}-03$ & 2.42 & $2.4843 \mathrm{e}-01$ & 0.93 \\
& 1089 & $2.71 \mathrm{e}+07$ & $1.2145 \mathrm{e}-01$ & 1.03 & $9.3189 \mathrm{e}-04$ & 2.41 & $1.2145 \mathrm{e}-01$ & 1.03 \\
& 4225 & $1.36 \mathrm{e}+07$ & $5.6633 \mathrm{e}-02$ & 1.10 & $2.2624 \mathrm{e}-04$ & 2.04 & $5.6633 \mathrm{e}-02$ & 1.10 \\
& 16641 & $6.80 \mathrm{e}+06$ & $2.7451 \mathrm{e}-02$ & 1.04 & $5.3728 \mathrm{e}-05$ & 2.07 & $2.7451 \mathrm{e}-02$ & 1.04 \\
\hline Mesh 3 & 81 & $1.08 \mathrm{e}+08$ & $6.7567 \mathrm{e}-01$ & - & $5.1494 \mathrm{e}-02$ & - & $6.7567 \mathrm{e}-01$ & - \\
& 289 & $5.41 \mathrm{e}+07$ & $2.8741 \mathrm{e}-01$ & 1.23 & $1.4971 \mathrm{e}-02$ & 1.78 & $2.8741 \mathrm{e}-01$ & 1.23 \\
& 1089 & $2.71 \mathrm{e}+07$ & $1.4203 \mathrm{e}-01$ & 1.01 & $3.7823 \mathrm{e}-03$ & 1.98 & $1.4203 \mathrm{e}-01$ & 1.01 \\
& 4225 & $1.36 \mathrm{e}+07$ & $6.9663 \mathrm{e}-02$ & 1.03 & $8.4736 \mathrm{e}-04$ & 2.16 & $6.9663 \mathrm{e}-02$ & 1.03 \\
& 16641 & $6.80 \mathrm{e}+06$ & $3.4586 \mathrm{e}-02$ & 1.01 & $1.9476 \mathrm{e}-04$ & 2.12 & $3.4586 \mathrm{e}-02$ & 1.01 \\
\hline
\end{tabular}

Example 4.2. In the second example from [9], we consider the problem (2.1) with variable coefficients, using the meshes presented in Fig. 1 in the case

$$
\begin{aligned}
& u=600 x y(1-x)(1-y)\left(x-\frac{1}{5}\right)\left(y-\frac{2}{5}\right)\left(y-\frac{3}{5}\right), \\
& K=10^{-7}\left(\begin{array}{cc}
1+x^{2} & x y \\
x y & 1+y^{2}
\end{array}\right) \\
& \beta=\left(\frac{1}{3}+10 y\left(x+y^{2}\right)^{4},-\frac{1}{2}-5\left(x+y^{2}\right)^{4}\right) .
\end{aligned}
$$

The right-hand side $f(\mathbf{x})$ is again determined by substituting $u$ into the Eq. (2.1).

The numerical results are presented in Table 3. The $H^{1}$ - and $L^{2}$-errors are optimal. Thus the stabilisation method is efficient for variable coefficient problems as well.

Example 4.3. Here we consider the VEM for the benchmark problem in [24]. The solution, the velocity field and the isotropic diffusion tensor are defined by

$$
u=\left(x-e^{2(x-1) / v}\right)\left(y^{2}-e^{3(y-1) / v}\right), \quad K=v \mathbf{I}, \quad \mathbf{b}=(2,3)^{T} .
$$

The right-hand side $f(\mathbf{x})$ is again determined by substituting $u$ into the Eq. (2.1). The term $v$ characterises thickness of the boundary layer in the top-right corner of the mesh. Here we choose $v=10^{-4}$ and solve the problem by using the meshes mentioned. The aim of these tests is to demonstrate that the stabilised VEM converges fast and has no oscillation outside of the boundary layer. The errors are computed in the domain $[0,0.8]^{2}$ and the convergence curves shown in Fig. 2, demonstrate the accuracy of the method. In Figs. 3-5 we display exact, stabilised and unstabilised solutions of the problem and note that for this convection-dominated problem, the stabilised VEM avoids numerical oscillations. 
Table 3: Example 4.2.

\begin{tabular}{||c|cc|cccccc||}
\hline Mesh & $D O F$ & Péclet & $\left\|u-u_{h}\right\|$ & order & $\left\|u-u_{h}\right\|_{0}$ & order & $\left\|u-u_{h}\right\|_{1}$ & order \\
\hline Mesh 1 & 81 & $2.08 \mathrm{e}+06$ & $1.7392 \mathrm{e}+00$ & - & $1.3543 \mathrm{e}-01$ & - & $1.7392 \mathrm{e}+00$ & - \\
& 289 & $1.04 \mathrm{e}+06$ & $8.7148 \mathrm{e}-01$ & 1.00 & $3.1276 \mathrm{e}-02$ & 2.11 & $8.7148 \mathrm{e}-01$ & 1.00 \\
& 1089 & $5.20 \mathrm{e}+05$ & $4.2169 \mathrm{e}-01$ & 1.05 & $2.9110 \mathrm{e}-03$ & 3.43 & $4.2169 \mathrm{e}-01$ & 1.05 \\
& 4225 & $2.60 \mathrm{e}+05$ & $2.1464 \mathrm{e}-01$ & 0.97 & $6.4068 \mathrm{e}-04$ & 2.18 & $2.1464 \mathrm{e}-01$ & 0.97 \\
& 16641 & $1.30 \mathrm{e}+05$ & $1.0210 \mathrm{e}-01$ & 1.07 & $1.5712 \mathrm{e}-04$ & 2.03 & $1.0210 \mathrm{e}-01$ & 1.07 \\
\hline Mesh 2 & 81 & $2.08 \mathrm{e}+06$ & $1.7041 \mathrm{e}+00$ & - & $1.0505 \mathrm{e}-01$ & - & $1.7041 \mathrm{e}+00$ & - \\
& 289 & $1.04 \mathrm{e}+06$ & $9.4071 \mathrm{e}-01$ & 0.86 & $3.4088 \mathrm{e}-02$ & 1.62 & $9.4071 \mathrm{e}-01$ & 0.86 \\
& 1089 & $5.20 \mathrm{e}+05$ & $4.6378 \mathrm{e}-01$ & 1.02 & $6.4644 \mathrm{e}-03$ & 2.40 & $4.6378 \mathrm{e}-01$ & 1.02 \\
& 4225 & $2.60 \mathrm{e}+05$ & $2.2970 \mathrm{e}-01$ & 1.01 & $1.2847 \mathrm{e}-03$ & 2.33 & $2.2970 \mathrm{e}-01$ & 1.01 \\
& 16641 & $1.30 \mathrm{e}+05$ & $1.1542 \mathrm{e}-01$ & 0.99 & $3.0172 \mathrm{e}-04$ & 2.09 & $1.1542 \mathrm{e}-01$ & 0.99 \\
\hline Mesh 3 & 81 & $2.08 \mathrm{e}+06$ & $1.5814 \mathrm{e}+00$ & - & $2.1597 \mathrm{e}-01$ & - & $1.5814 \mathrm{e}+00$ & - \\
& 289 & $1.04 \mathrm{e}+06$ & $1.0120 \mathrm{e}+00$ & 0.64 & $8.2123 \mathrm{e}-02$ & 1.40 & $1.0120 \mathrm{e}+00$ & 0.64 \\
& 1089 & $5.20 \mathrm{e}+05$ & $4.8154 \mathrm{e}-01$ & 1.07 & $2.1496 \mathrm{e}-02$ & 1.93 & $4.8154 \mathrm{e}-01$ & 1.07 \\
& 4225 & $2.60 \mathrm{e}+05$ & $2.2346 \mathrm{e}-01$ & 1.11 & $4.1554 \mathrm{e}-03$ & 2.37 & $2.2346 \mathrm{e}-01$ & 1.11 \\
& 16641 & $1.30 \mathrm{e}+05$ & $1.0950 \mathrm{e}-01$ & 1.02 & $7.0241 \mathrm{e}-04$ & 2.56 & $1.0950 \mathrm{e}-01$ & 1.02 \\
\hline
\end{tabular}

Example 4.4. We consider the stabilised VEM for a problem with exponential boundary layers for the benchmark problem in [21]. The convection field $\mathbf{b}=(1 / 2, \sqrt{3} / 2)^{T}$. The exact solution and diffusion field are given by

$$
\begin{aligned}
& u=\left(\frac{x^{2}}{2 a_{1}}+\frac{v x}{a_{1}^{2}}+\left(\frac{1}{2 a_{1}}+\frac{v}{a_{1}^{2}}\right) \frac{e^{-a_{1} / v}-e^{-a_{1} / v}(1-x)}{1-e^{-a_{1} / v}}\right) \\
& \times\left(\frac{y^{2}}{2 a_{2}}+\frac{v y}{a_{2}^{2}}+\left(\frac{1}{2 a_{2}}+\frac{v}{a_{2}^{2}}\right) \frac{e^{-a_{2} / v}-e^{-a_{2} / v}(1-y)}{1-e^{-a_{2} / v}}\right), \\
& K=\nu \mathbf{I} .
\end{aligned}
$$

The term $v$ characterises the thickness of the boundary layer in the top-right corner of the mesh. We choose $v=10^{-9}$ and compute the errors in the domain $[0,0.8]^{2}$. The numerical convergence rate shown in Fig. 6 is consistent with the theoretical results. Figs. 79 show that for this convection-dominated problem the stabilised method avoids numerical oscillation.

\section{Conclusions}

We study a novel streamline diffusion form of virtual element method for convectiondominated diffusion problems. Unlike the standard VEM, the stabilisation scheme can efficiently avoid nonphysical oscillations. We also prove the well-posedness of the problem and provide error estimates for the stabilisation scheme. Numerical examples show the stability of the method for very large Péclet numbers and its applicability to boundary layer problem. 


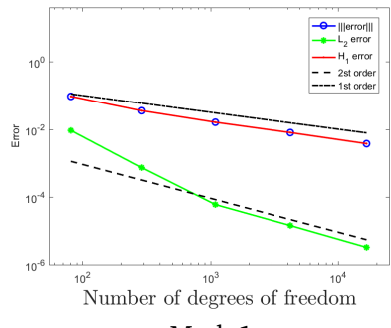

Mesh 1

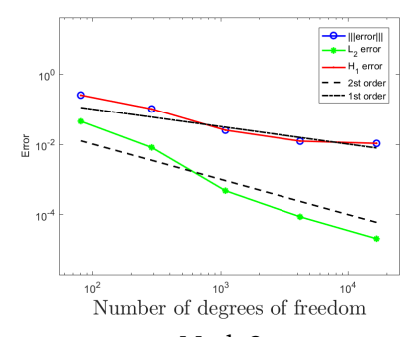

Mesh 2

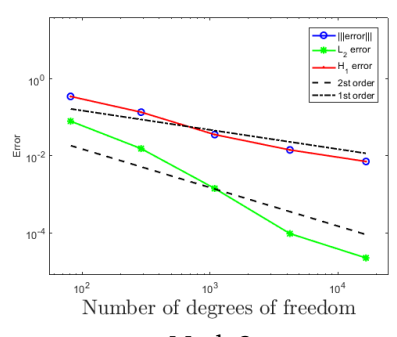

Mesh 3

Figure 2: Convergence curves on Mesh 1 - Mesh 3 in Example 4.3.

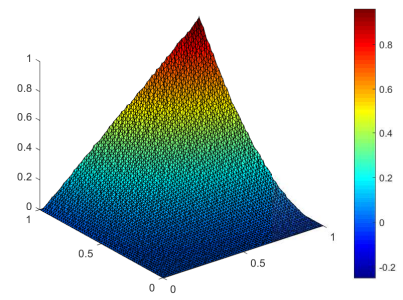

Exact solution

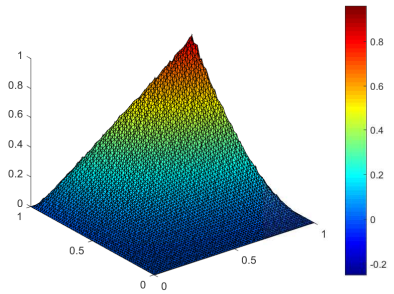

Stabilised solution

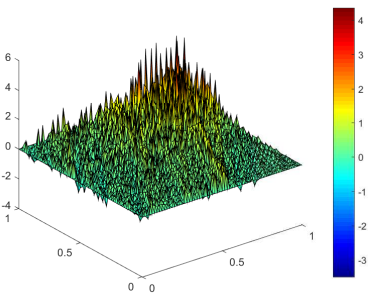

Unstabilised solution

Figure 3: Example 4.3. Exact, stabilised and unstabilised solutions on the Mesh 1 with the Péclet number $5.63 \times 10^{3}$.

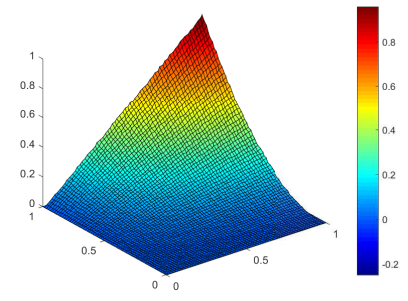

Exact solution

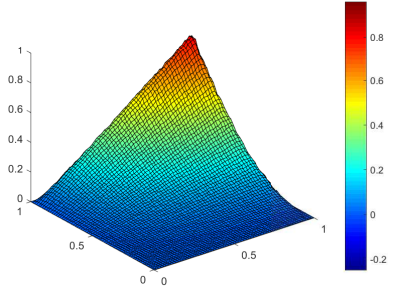

Stabilised solution

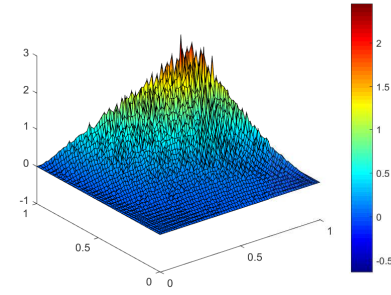

Unstabilised solution

Figure 4: Example 4.3. Exact, stabilised and unstabilised solutions on the Mesh 2 with the Péclet number.

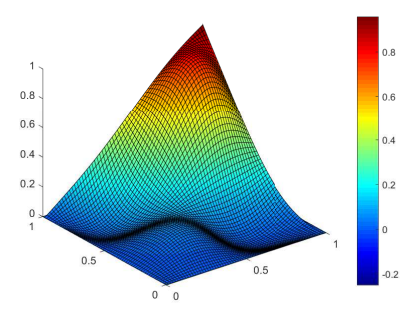

Exact solution

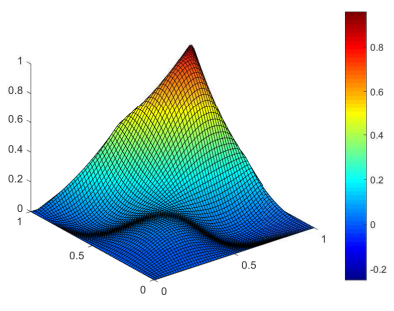

Stabilised solution

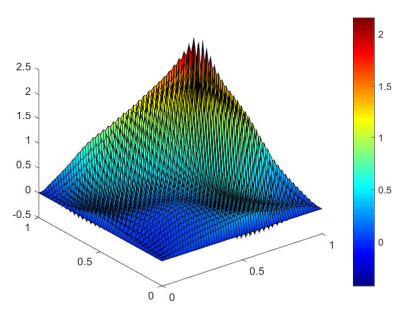

Unstabilised solution

Figure 5: Example 4.3. Exact, stabilised and unstabilised solutions on the Mesh 3 with the Péclet number $5.63 \times 10^{3}$. 


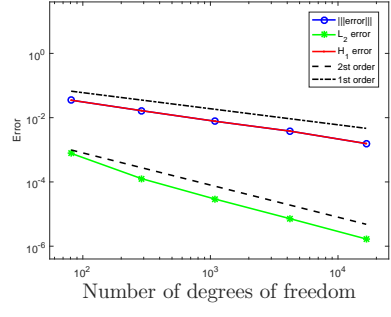

Mesh 1

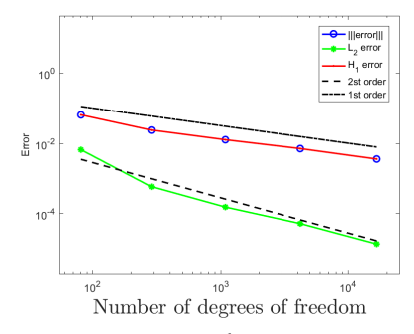

Mesh 2

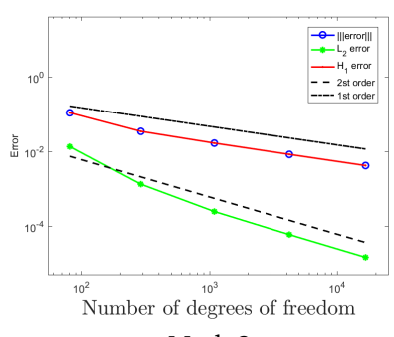

Mesh 3

Figure 6: Example 4.4. Convergence curves.

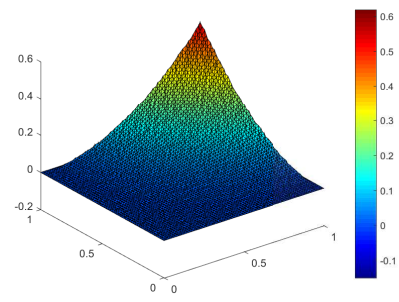

Exact solution

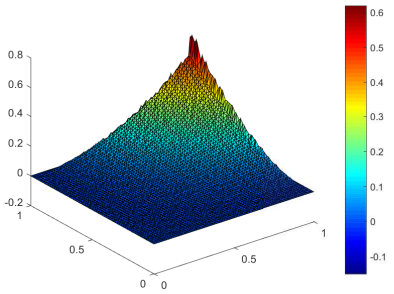

Stabilised solution

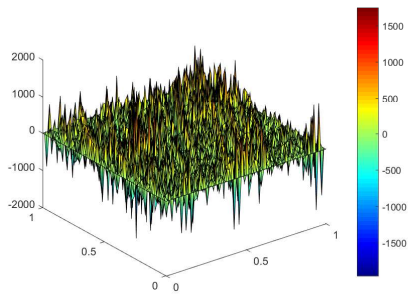

Unstabilised solution

Figure 7: Example 4.4. Exact, stabilised, unstabilised solutions on Mesh 1 with the Péclet numbers $1.56 \times 10^{7}$.

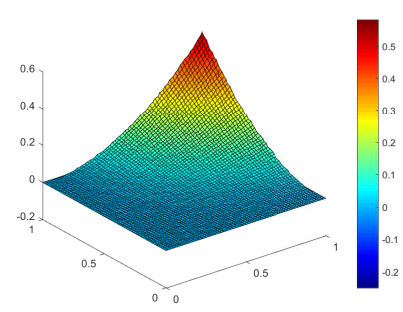

Exact solution

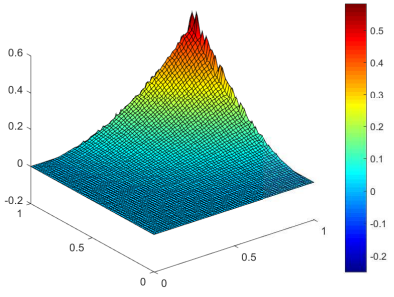

Stabilised solution

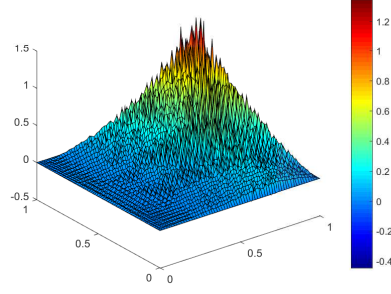

Unstabilised solution

Figure 8: Example 4.4. Exact, stabilised, unstabilised solutions on Mesh 2 with the Péclet numbers $1.56 \times 10^{7}$.

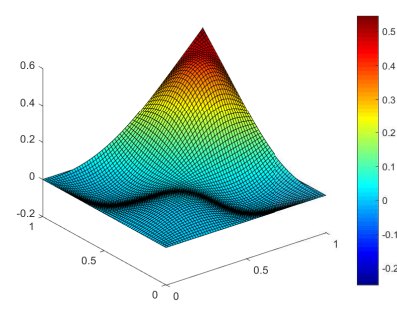

Exact solution

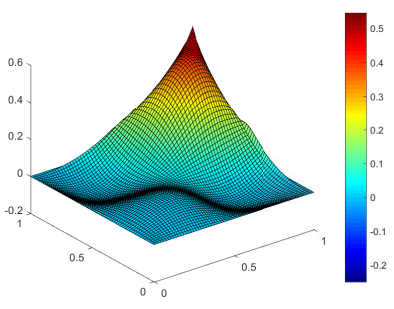

Stabilised solution

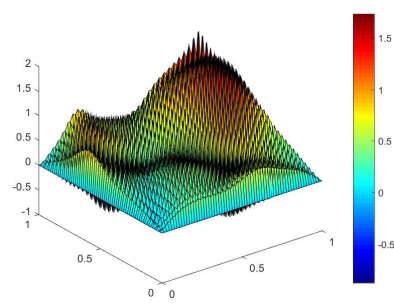

Unstabilised solution

Figure 9: Example 4.4. Exact, stabilised, unstabilised solutions on Mesh 3 with the Péclet numbers $1.56 \times 10^{7}$. 


\section{Acknowledgments}

The authors would like to thank Professor Chunxiong Zheng for research assistance, the anonymous referees and Professor Victor D. Didenko for valuable comments and suggestions which helped to improve the quality of this paper.

This work was supported in parts by the Research Fund of the Key Laboratory of Xinjiang Province (No. 2017D04030) and by the NSF of China (Nos. 11671345, 11362021). The second author was also supported by the "Postdoctoral International Exchange Program".

\section{References}

[1] B. Ahmad, A. Alsaedi, F. Brezzi, L.D. Marini and A. Russo, Equivalent projectors for virtual element methods, Math. Models Methods Appl. Sci. 66, 376-391 (2013).

[2] P.F. Antonietti, L. Beirão da Veiga, D. Mora and M. Verani, A stream virtual element formulation of the Stokes problem on polygonal meshes, SIAM J. Numer. Anal. 52, 386-404 (2014).

[3] L. Beirão da Veiga, F. Brezzi, A. Cangiani, G. Manzini, L.D. Marini and A. Russo, Basic principles of virtual element methods, Math. Models Methods Appl. Sci. 23, 199-214 (2013).

[4] L. Beirão Da Veiga, F. Brezzi, L.D. Marini and A. Russo, The hitchhiker's guide to the virtual element method, Math. Models Methods Appl. Sci. 24, 1541-1573 (2014).

[5] L. Beirão da Veiga, F. Brezzi, L.D. Marini and A. Russo. Virtual element method for general second-order elliptic problems on polygonal meshes, Math. Models Methods Appl. Sci. 26, 7589 (2016).

[6] L. Beirão da Veiga, C. Lovadina and D. Mora. A virtual element method for elastic and inelastic problems on polytope meshes, Comput. Methods Appl. Mech. Engrg. 295, 327-346 (2015).

[7] L. Beirão da Veiga, C. Lovadina and G. Vacca, Virtual elements for the Navier-Stokes problem on polygonal meshes, SIAM J. Numer. Anal. 56, 1210-1242 (2018).

[8] M.F. Benedetto, S. Berrone, A. Borio and S. Pieraccini. A hybrid mortar virtual element method for discrete fracture network simulations, J. Comput. Phys. 306, 148-166 (2016).

[9] M.F. Benedetto, S. Berrone, A. Borio, S. Pieraccini and S. Scialò, Order preserving SUPG stabilization for the virtual element formulation of advection-diffusion problems, Comput. Methods Appl. Mech. Engrg. 311, 18-40 (2016).

[10] S. Berrone and A. Borio, Orthogonal polynomials in badly shaped polygonal elements for the virtual element method, Finite Elem. Anal. Des. 129, 14-31 (2017).

[11] S. Berrone, A. Borio and G. Manzini, SUPG stabilization for the nonconforming virtual element method for advection-diffusion-reaction equations, Comput. Methods Appl. Mech. Engrg. 340, 500-529 (2018).

[12] F. Brezzi, L.P. Franca and A. Russo, Further considerations on residual-free bubbles for advectivediffusive equations, Comput. Methods Appl. Mech. Engrg. 166, 25-33 (1998).

[13] F. Brezzi, K. Lipnikov and V. Simoncini, A family of mimetic finite difference methods on polygonal and polyhedral meshes, Math. Models Methods Appl. Sci. 15, 1533-1551 (2005).

[14] F. Brezzi and L.D. Marini, Virtual element methods for plate bending problems, Comput. Methods Appl. Mech. Engrg. 253, 455-462 (2013).

[15] A.N. Brooks and T.J.R. Hughes, Streamline upwind/Petrov-Galerkin formulations for convection dominated flows with particular emphasis on the incompressible Navier-Stokes equations, Comput. Methods Appl. Mech. Engrg. 32,199-259 (1982). 
[16] A. Cangiani, G. Manzini and O. Sutton, The conforming virtual element method for the convection-diffusion-reaction equation with variable coeffcients, United States: N. p. Web. doi:10.2172/1159207 (2014).

[17] H.Y. Duan, P.W. Hsieh, R.C.E. Tan and S.Y. Yang, Analysis of a new stabilized finite element method for the reaction-diffusion-convection equations with a large reaction coefficient, Comput. Methods Appl. Mech. Engrg. 247-248, 15-36 (2012).

[18] L.P. Franca, S.L. Frey and T.J.R. Hughes, Stabilized finite element methods: I. Application to the advective-diffusive model, Comput. Methods Appl. Mech. Engrg. 95, 253-276(1992).

[19] L.P. Franca and L. Tobiska, Stability of the residual free bubble method for bilinear finite elements on rectangular grids, IMA J. Numer. Anal. 22, 73-87 (2002).

[20] G. Fu, W. Qiu and W. Zhang, An analysis of HDG methods for convection-dominated diffusion problems, ESAIM Math. Model. Numer. Anal. 49, 225-256 (2015).

[21] P.W. Hsieh and S.Y. Yang, A new stabilized linear finite element method for solving reactionconvection-diffusion equations, Comput. Methods Appl. Mech. Engrg. 307, 362-382 (2016).

[22] N. Li, H.Y. Su, D.W. Gui and X.L. Feng, Multiquadric RBF-FD method for the convectiondominated diffusion problems base on Shishkin nodes, Int. J. Heat. Mass. Tran. 118, 734-745 (2018).

[23] K. Lipnikov, G. Manzini and M. Shashkov, Mimetic finite difference method, J. Comput. Phys. 257, 1163-1227 (2014).

[24] K. Lipnikov, D. Svyatskiy and Y. Vassilevski, A monotone finite volume method for advectiondiffusion equations on unstructured polygonal meshes, J. Comput. Phys. 229, 4017-4032 (2010).

[25] G. Manzini and A. Russo, A finite volume method for advection-diffusion problems in convectiondominated regimes, Comput. Methods Appl. Mech. Engrg. 197, 1242-1261 (2008).

[26] L.Z. Qian, X.L. Feng and Y.N. He, The characteristic finite difference streamline diffusion method for convection-dominated diffusion problems, Comput. Methods Appl. Math. Model. 36, 561572 (2012).

[27] Z.Y. Si, X.L. Feng and A. Abduwali, The semi-discrete streamline diffusion finite element method for time-dependented convection-diffusion problems, Appl. Math. Comput. 202, 771779 (2008).

[28] N. Sukumar and A. Tabarraei, Conforming polygonal finite elements, Internat. J. Numer. Methods Engrg. 61, 2045-2066 (2004).

[29] F. Wang and H. Wei, Virtual element method for simplified friction problem, Appl. Math. Lett. 85, 125-131 (2018). 